MKPH-T-12-09

HIM-2012-01

\title{
Factorizing the hard and soft spectator scattering contributions for the nucleon form factor $F_{1}$ at large $Q^{2}$
}

\author{
N. Kivel* \\ Institut für Kernphysik, Johannes Gutenberg-Universität, D-55099 Mainz, Germany and \\ Helmholtz Institut Mainz, Johannes Gutenberg-Universität, D-55099 Mainz, Germany
}

In [1] we suggested the factorization formula for the nucleon form factors which consist of the sum of two contributions describing the hard and soft spectator scattering, and we provided a description of the soft rescattering contribution for the $\mathrm{FF} F_{1}$ in terms of convolution integrals of the hard and hard-collinear coefficient functions with the appropriate soft matrix elements.

In present paper we investigate the soft spectator scattering contribution for the FF $F_{1}$. We focus our attention on factorization of the hard-collinear scale $\sim Q \Lambda$ corresponding to transition from SCET-I to SCET-II. We compute the leading order jet functions and find that the convolution integrals over the soft fractions are logarithmically divergent. This divergency is the consequence of the boost invariance and does not depend on the model of the soft correlation function describing the soft spectator quarks. Using as example a two-loop diagram we demonstrated that such a divergency corresponds to the overlap of the soft and collinear regions. As a result one obtains large rapidity logarithm which must be included in the correct factorization formalism.

We conclude that a consistent description of the factorization for $F_{1}$ implies the end-point collinear divergencies in the hard and soft spectator contributions, i.e. convolution integrals with respect to collinear fractions are not well-defined. Such scenario can only be realized when the twist-3 nucleon distribution amplitude has specific end-point behavior which differs from one expected from the evolution of the nucleon distribution amplitude. Such behavior leads to the violation of the collinear factorization for the hard spectator scattering contribution. We suggest that the soft spectator scattering and chiral symmetry breaking provide the mechanism responsible for the violation of collinear factorization in case of form factor $F_{1}$.

In spite of complexities of the SCET factorization it can be very useful for a phenomenological analysis of hard exclusive reactions. The basis for such approach is provided by universality of the SCET-I form factors which can appear in different hard processes. We show that using, so-called, physical subtraction scheme SCET factorization in some cases allows to perform the systematical analysis of the hadronic processes in the range of moderate values of $Q^{2} \sim 5-20 \mathrm{GeV}^{2}$ where the hard collinear scale $\sim Q \Lambda$ is still not large

\section{INTRODUCTION}

A substantial progress of the experimental studies of the nucleon form factors (FFs) has been achieved during the last decade. The polarization transfer method allowed one to measure accurately the proton FFs up to momentum transfer $Q^{2} \simeq 8.5 \mathrm{GeV}^{2}[2[5]$, for recent reviews see, e.g., Refs. [6 8] ]. It also opened a possibility for systematic studies of the FFs at large space-like $Q^{2}$ region in the near future at the Jlab $12 \mathrm{GeV}$ upgraded facility see, e.g., [9]. At the same time the PANDA Collaboration at GSI is planning to carry out precise measurements of the proton FFs at large time-like momentum transfers, up to around $20 \mathrm{GeV}^{2}$, using the annihilation process $p+\bar{p} \rightarrow e^{+}+e^{-}[10,11]$. These experiments will provide us with new information on the FF behavior in the region of large momentum transfers that also provides a strong motivation for the theoretical studies of the large- $Q$ behavior of the nucleon FFs.

It is known for a long time that in the case of nucleon FFs the soft spectator scattering is not suppressed by inverse powers of large $Q$ [12 15]. Moreover, results of different phenomenological approaches 16 22] allow one to conclude that in the region of moderate $Q^{2} \sim 5-15 \mathrm{GeV}^{2}$ such mechanism plays the crucial

* On leave of absence from Petersburg Nuclear Physics Institute, Gatchina, 188350, Russia 
role in order to obtain correct description of the FFs data and also other hard processes with baryons see, e.g., 23 26]. A systematic QCD approach for description of such soft mechanism is needed in order to develop a model-independent formalism for description of existing and future experiments.

In [1] the contribution of the soft spectator scattering has been included into factorization scheme using the formalism of the soft collinear effective theory (SCET) 27 32]. The important feature in the description of the soft-overlap contribution is the presence of the hard-collinear scale $\sim Q \Lambda$. In order to take into account the soft rescattering mechanism one uses the two-step matching technique developed in SCET. Following from the leading-logarithmic analysis we suggested that the tentative factorization formula for the Dirac FF $F_{1}$ can be written as a sum of the hard and soft rescattering contributions:

$$
F_{1} \simeq F_{1}^{(h)}+F_{1}^{(s)}
$$

where the hard rescattering part $F_{1}^{(h)}$ is described by convolution of the hard coefficient function $\mathbf{H}$ with the nucleon distribution amplitudes (DAs) $\boldsymbol{\Psi}$ :

$$
F_{1}^{(h)}=\int D x_{i} \int D y_{i} \mathbf{\Psi}\left(y_{i}\right) \mathbf{H}\left(x_{i}, y_{i} \mid Q\right) \mathbf{\Psi}\left(x_{i}\right) \equiv \boldsymbol{\Psi} * \mathbf{H} * \mathbf{\Psi},
$$

while the soft contribution $F_{1}^{(s)}$ has the same scaling behavior $\sim 1 / Q^{4}$ as the hard spectator term $F_{1}^{(h)}$ and can be presented in the following form :

$$
F_{1}^{(s)} \simeq C_{A}(Q) \int D y_{i} \boldsymbol{\Psi}\left(y_{i}\right) \int_{0}^{\infty} d \omega_{1} d \omega_{2} \mathbf{J}^{\prime}\left(y_{i}, \omega_{i} Q\right) \int D x_{i} \boldsymbol{\Psi}\left(x_{i}\right) \int_{0}^{\infty} d \nu_{1} d \nu_{2} \mathbf{J}\left(x_{i}, \nu_{i} Q\right) \boldsymbol{S}\left(\omega_{i}, \nu_{i}\right) .
$$

This formula can be interpreted graphically as reduced diagram in Fig 1 The hard subprocesses in the soft spectator contribution are described by the hard coefficient function $C_{A}$ and two hard-collinear jet functions $\mathbf{J}, \mathbf{J}^{\prime}$. They describe the parton scattering with the hard and hard-collinear momenta, respectively. The non-perturbative DA $\boldsymbol{\Psi}$ and soft correlation function (SCF) $\boldsymbol{S}$ describe the long distance scattering of collinear and soft modes. The convolution integrals in Eq. (3) are performed with respect to the collinear fractions $x_{i}$ and $y_{i}$, and with respect to the soft spectator fractions $\omega_{i}, \nu_{i} \sim \Lambda$.

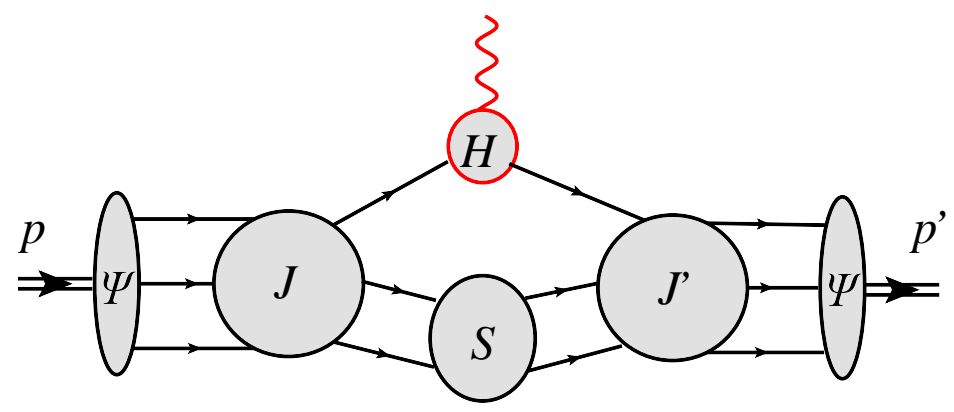

FIG. 1: Interpretation of the soft rescattering as a reduced diagram

In [1] we discussed the matching of electro-magnetic current onto SCET-I operator, calculation hard coefficient function $C_{A}$ and resummation of Sudakov logarithms. The structure of the factorization formula (3) was considered only qualitatively. In the current publication we present more detailed consideration of this contribution. We shall carefully consider the matching of SCET-I to SCET-II and check the validity of the Eq.(3).

In 11 we assumed the existence of all convolution integrals with respect to the collinear and soft fractions in (3). Such assumption is motivated by the following observation. First, one can easily observe that collinear convolution integrals in both contributions (2) and (3) have a similar structure. Second, if one uses phenomenological models of the nucleon DAs existing in literature then the collinear integrals are well defined. After that we expected that the integral over the soft fractions must be also well defined. However in this work we show using some general model-independent arguments that the convolution with respect to the soft fractions in (3) is divergent. This divergence can be represented as scaleless 
logarithmic integral over one of the fractions: $\sim \int_{0}^{\infty} d \omega_{1} / \omega_{1}$. Obviously, such integral has problems in both ultraviolet (UV) and infrared (IR) regions. The divergence in UV-limit is a signal that here one faces with the well known problem: overlapping of the soft and collinear modes.

In order to clarify the situation we perform more careful analysis of the factorization (1) in the perturbation theory using perturbative interpretations for the DAs and soft correlation function. It turns out that in the theory with massive quarks the collinear convolution integrals in (21) and (3) are also divergent. This divergency arises due to the overlap of the soft and collinear sectors. We demonstrate that there is large rapidity logarithm $\sim \ln Q / \Lambda$ which can be computed due to the overlap of collinear and soft regions associated with (2) and (3). This is exactly the logarithm which was computed in [12]. In order to compute (2) and (3) unambiguously one has to define a certain prescription which allows one to separate the collinear and soft sectors and to avoid a double counting. In the case of perturbation theory such separation can be carried out using the the idea of subtractions discussed in QCD for the Sudakov FF [33] or, similar technique in SCET known as zero-bin subtraction method [34]. We obtain that the perturbative calculations of the studied diagram is in agreement with the factorization formula (2). However generalization of the perturbative results to realistic case faces with certain difficulties and the description of the hard-collinear factorization even for $F_{1}$ remains challenging.

The overlap of the collinear and soft regions imposes also a qualitative idea about the end-point behavior of the twist-3 nucleon DA. We expect that the end-point behavior of nucleon DA at low normalization differs from the behavior of the asymptotic DA $\boldsymbol{\Psi}_{a s}\left(x_{i}\right) \sim x_{1} x_{2} x_{3}$. Usually the models of DA always vanish quadratically when the fractions $x_{i}$ are small. But DA should have a different behavior in order to produce the end-point singularity in the convolution integrals. We show that example of such behavior can be obtained even in the perturbation theory with massive quarks: for instance, if $x_{1,2} \rightarrow 0$ and $x_{1} \sim x_{2}$, then the perturbative DA vanishes only linearly as $\mathbf{\Psi}\left(x_{1}, x_{2}, x_{3}\right) \sim x_{1,2}$. This already leads to the end-point singularity in the collinear convolution integrals. The presence of soft quark mass in this calculation is necessary. The combination of two possibilities: soft spectator scattering and explicit chiral symmetry breaking due to the quark mass leads to the violation of the collinear factorization in case of helicity conserving Dirac form factor. Taking into account the dynamical chiral symmetry breaking in QCD we expect a realization of the similar scenario for the nonperturbative nucleon DA.

Our presentation is organized as follows. In Sec. II we describe in details the soft rescattering contribution $F_{1}^{(s)}$ defined by Eq.(3), present the analytic results for the leading-order jet functions and discuss the divergency of the soft convolution integrals. Sec. III is dedicated to the analysis of the overlap between the soft and collinear modes. Using two-loop QCD diagram we calculate the large rapidity logarithm and perform the interpretation of the obtained results in terms of hard $F_{1}^{(h)}$ and soft $F_{1}^{(s)}$ contributions. Generalizing these observations we suggest that collinear integrals in the factorization formulas for $F_{1}^{(h)}$ and $F_{1}^{(s)}$ are also divergent and therefore the nucleon DA must have a specific end-point behavior. In Sec.IV we discuss the application of the SCET factorization for the phenomenological analysis at moderate values of $Q^{2}$. In Sec.V, we summarize our results.

\section{SOFT SPECTATOR SCATTERING CONTRIBUTION}

We begin our discussion from the description of the soft rescattering contribution. All notations are the same as in [1] and are briefly described in Appendix A. For simplicity we consider proton as the target nucleon. In order to describe $F_{1}^{(s)}$ we need to define two nonperturbative functions: proton DA $\Psi\left(x_{i}\right)$ and soft correlation function $(\mathrm{SCF}) \boldsymbol{S}\left(\omega_{i}, \nu_{i}\right)$.

The proton DA is a well-known object defined as:

$$
4\left\langle 0\left|W_{c}^{\dagger}\left[\lambda_{1} n\right] \xi_{\alpha_{1}}^{u} W_{c}^{\dagger} \xi_{\alpha_{2}}^{u}\left[\lambda_{2} n\right] W_{c}^{\dagger} \xi_{\alpha_{3}}^{d}\left[\lambda_{3} n\right]\right| p\right\rangle=\frac{\varepsilon^{i j k}}{3 !} \int D x_{i} e^{-i p_{+}\left(\sum x_{i} \lambda_{i}\right)} \Psi_{\boldsymbol{\alpha}}\left(x_{i}\right),
$$

where the measure reads $D x_{i}=d x_{1} d x_{2} d x_{3} \delta\left(1-x_{1}-x_{2}-x_{3}\right), \varepsilon^{i j k}$ is the color factor, the multiindex $\boldsymbol{\alpha} \equiv \alpha_{1} \alpha_{2} \alpha_{3}$, and the index "c" denotes in SCET-II the collinear fields :

$$
W_{c}^{\dagger}[x] \xi_{\alpha}^{u} \equiv \overline{\mathrm{P}} \exp \left\{-i g \int_{-\infty}^{0} d t\left(n \cdot A_{c}\right)(x+t n)\right\}\left[\frac{\not h n}{4} u_{c}(x)\right]_{\alpha},
$$


The function $\boldsymbol{\Psi}_{\boldsymbol{\alpha}}\left(x_{i}\right)$ can be further represented as

$$
\begin{aligned}
& \Psi_{\boldsymbol{\alpha}}\left(x_{i}\right)=V\left(x_{i}\right) p_{+}\left[\frac{1}{2} \hbar C\right]_{\alpha_{1} \alpha_{2}}\left[\gamma_{5} \frac{\not h \mu h}{4} N\right]_{\alpha_{3}}+A\left(x_{i}\right) p_{+}\left[\frac{1}{2} \not{\hbar} \gamma_{5} C\right]_{\alpha_{1} \alpha_{2}}\left[\frac{\not h h}{4} N\right]_{\alpha_{3}} \\
& +T\left(x_{i}\right) p_{+}\left[\frac{1}{2} \not h \gamma_{\perp} C\right]_{\alpha_{1} \alpha_{2}}\left[\gamma^{\perp} \gamma_{5} \frac{\not h \not h}{4} N\right]_{\alpha_{3}},
\end{aligned}
$$

where $C$ is the charge conjugate matrix $C: C \gamma_{\mu} C=\gamma_{\mu}^{T}$. The scalar functions $V, A, T$ depend on the collinear fractions $x_{i}$ and from the factorization scale which is not shown for simplicity. Alternatively, these functions can be represented through the one twist-3 DA $\varphi_{N}\left(x_{i}\right)$ [35]:

$$
\begin{aligned}
& V\left(x_{1}, x_{2}, x_{3}\right) \equiv V(1,2,3)=\frac{1}{2}\left(\varphi_{N}(1,2,3)+\varphi_{N}(2,1,3)\right), \\
& A(1,2,3)=-\frac{1}{2}\left(\varphi_{N}(1,2,3)-\varphi_{N}(2,1,3)\right), \\
& T(1,2,3)=\frac{1}{2}\left(\varphi_{N}(1,3,2)+\varphi_{N}(2,3,1)\right) .
\end{aligned}
$$

In many applications $\varphi_{N}\left(x_{i}\right)$ usually is approximated by a few polynomials in $x_{i}$ with unknown coefficients. The polynomials represent the eigenfunctions of the evolution kernels and naturally arise from the solution of the evolution equation ( see,e.g., [36, 37] and references there in). For example, the one of most popular parametrizations for $\varphi_{N}\left(x_{i}\right)$ can be represented as follows [35]:

$$
\varphi_{N}\left(x_{i}\right) \simeq 120 x_{1} x_{2} x_{3} f_{N}\left(1+c_{10}\left(x_{1}-2 x_{2}+x_{3}\right)+c_{11}\left(x_{1}-x_{3}\right)\right)
$$

The values of the nonperturbative constants $f_{N}, c_{i}$ are not important for our further discussion and we will not provide their numerical estimates.

A parametrization such as in Eq.(10) always fulfills one important property: it vanishes quadratically at the boundary where the fractions are close to zero, for instance if $x_{1} \sim x_{2} \rightarrow 0$ one obtains

$$
\varphi_{N}\left(x_{1}, x_{2}, x_{3}\right) \sim x_{1} x_{2} .
$$

Such behavior is an important requirement which ensures the existence of the convolution integrals in the factorization formula (2) because the hard coefficient functions, as a rule, have the end-point singularities $\sim 1 / x_{1} x_{2}$. The end-point behavior (11) can be associated with the corresponding behavior of the evolution kernel. Performing the expansion of the DA $\varphi_{N}$ in terms of the eigenfunctions of the evolution kernel and neglecting by the higher order harmonics one always obtains the model which vanishes at the boundary (11). However such approach is consistent only if one assumes an appropriate convergence of the conformal expansion at a given normalization point.

The second non-perturbative input is the SCF $\boldsymbol{S}$ introduced in Eq.(3). In SCET it is constructed from the soft quark and gluon fields $q$ and $A_{s}$, respectively. The gluon fields enter only in the form of Wilson lines such as

$$
S_{\bar{n}}(x)=\mathrm{P} \exp \left\{i g \int_{-\infty}^{0} d s \bar{n} \cdot A_{s}(x+s \bar{n})\right\}, Y_{n}^{\dagger}(x)=\mathrm{P} \exp \left\{i g \int_{0}^{\infty} d s n \cdot A_{s}(x+s n)\right\}
$$

The definition of $\boldsymbol{S}$ given in [1] implies that it is a tensor with respect to Dirac indices. It is convenient to rewrite it in terms of scalar functions. This can be done with the help of Fierz transformation so that the result reads:

$$
\boldsymbol{S}\left(\omega_{i}, \nu_{i}\right)=\frac{1}{8} C \not h \otimes \frac{1}{8} \not h C S_{V}\left(\omega_{i}, \nu_{i}\right)+\frac{1}{8} C \not h \gamma_{5} \otimes \frac{1}{8} \not h \gamma_{5} C S_{A}\left(\omega_{i}, \nu_{i}\right)+\frac{1}{4} C \not h \gamma_{\perp}^{\mu} \otimes \frac{1}{4} \not h \gamma_{\perp}^{\nu} C S_{T}^{\mu \nu}\left(\omega_{i}, \nu_{i}\right) .
$$

The scalar functions $S_{V, A, T}$ defined as

$$
S_{X}\left(\omega_{i}, \nu_{i}\right)=\int \frac{d \eta_{1}}{2 \pi} \int \frac{d \eta_{2}}{2 \pi} e^{-i \eta_{1} \nu_{1}-i \eta_{2} \nu_{2}} \int \frac{d \lambda_{1}}{2 \pi} \int \frac{d \lambda_{2}}{2 \pi} e^{i \lambda_{1} \omega_{1}+i \lambda_{2} \omega_{2}}\left\langle 0\left|O_{X}\left(\eta_{i}, \lambda_{i}\right)\right| 0\right\rangle,
$$



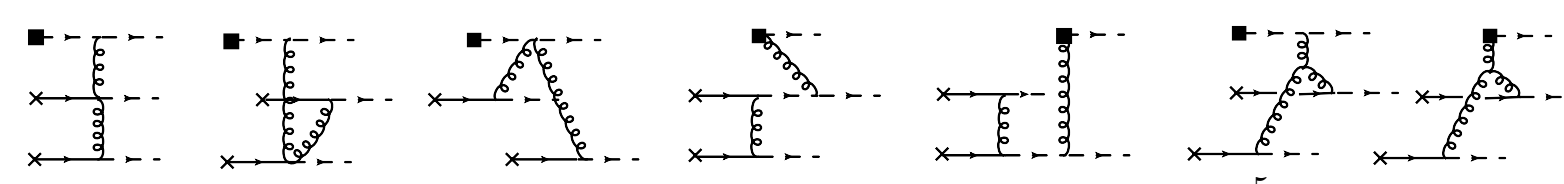

with the operators

$$
\begin{aligned}
O_{X}\left(\eta_{i}, \lambda_{i}\right) & =\varepsilon^{i^{\prime} j^{\prime} k^{\prime}}\left[Y_{n}^{\dagger}(0)\right]^{i^{\prime} l}\left[Y_{n}^{\dagger} q\left(\lambda_{1} n\right)\right]^{j^{\prime}} C \Gamma_{X}\left[Y_{n}^{\dagger} q\left(\lambda_{2} n\right)\right]^{k^{\prime}} \\
& \times \varepsilon^{i j k}\left[S_{\bar{n}}(0)\right]^{l i}\left[\bar{q} S_{\bar{n}}\left(\eta_{1} \bar{n}\right)\right]^{j} \bar{\Gamma}_{X} C\left[\bar{q} S_{\bar{n}}\left(\eta_{2} \bar{n}\right)\right]^{k}
\end{aligned}
$$

where

$$
\left\{\Gamma_{X} \otimes \bar{\Gamma}_{X}\right\}_{X=V, A, T}=\left\{\not h \otimes \not h, \not h \gamma_{5} \otimes \not h \gamma_{5}, \not h \gamma_{\perp} \otimes \not h \gamma_{\perp}\right\}
$$

The flavor structure of the SCFs $S_{X}$ is defined by the flavor structure of the proton: it can be described as either $u u$ - or $u d$-combinations. Below we will show that the tensor component $S_{T}$ does not contribute to $F_{1}$ and hence we can conclude that the matrix $\boldsymbol{S}\left(\omega_{i}, \nu_{i}\right)$ is presented by four scalar functions: $S_{A, V}^{\text {uu,ud }}\left(\omega_{i}, \nu_{i}\right)$. Indeed, SCFs also depend on the factorization scale, which is not shown for simplicity.

One has to keep in mind that the arguments $\omega_{i}$ and $\nu_{i}$ (soft fractions) are of order $\Lambda$. Obviously, these variables can be associated with the light-cone projections of the soft spectators momenta which are defined to be positive. For instance, the leading order calculation of the SCF $S_{V}^{\mathrm{ud}}\left(\omega_{i}, \nu_{i}\right)$ in perturbation theory yields (see details in Appendix B)

$$
\left[S_{V}^{\mathrm{ud}}\left(\omega_{i}, \nu_{i}\right)\right]_{\mathrm{LO}}=\frac{3 m^{2}}{16 \pi^{6}} \theta\left(\nu_{i}>0\right) \theta\left(\omega_{i}>0\right) \theta\left(\omega_{1} \nu_{1}>m^{2}\right) \theta\left(\omega_{2} \nu_{2}>m^{2}\right)
$$

where $m$ denotes the mass of soft quarks. Notice that $S_{V, A}$ is proportional to the square of mass $m^{2}$ which arises from the numerators of the soft quark propagators and this fact, as we will see later, has an important consequence.

The last elements in Eq.(3) which one has to introduce are jet functions $\mathbf{J}$ and $\mathbf{J}^{\prime}$. These are hardcollinear coefficient functions which can be computed in pQCD if the hard-collinear scale $Q \Lambda$ is quite large. They appear in the matching of hard-collinear modes onto collinear and soft fields in SCET-II. Jet functions can be computed from the $T$-products which schematically can be written as [1] :

$$
\begin{aligned}
& T\left(\bar{\xi}_{h c}^{\prime} W^{\prime} e^{i \mathcal{L}_{\text {SCET-I }}^{(n)}}\right) \simeq T\left(\bar{\xi}_{h c}^{\prime} W^{\prime}, i \mathcal{L}_{\xi^{\prime} q}^{(1)}, i \mathcal{L}_{\xi^{\prime} q}^{(1)}, i \mathcal{L}_{\xi^{\prime} \xi^{\prime}}^{(0)}\right)=\bar{\xi}_{c}^{\prime} \bar{\xi}_{c}^{\prime} \bar{\xi}_{c}^{\prime} * \mathbf{J}_{L O}^{\prime} * q q, \\
& T\left(W^{\dagger} \xi_{h c} e^{i \mathcal{L}_{\text {SCET-I }}^{(\bar{n})}}\right) \simeq T\left(W^{\dagger} \xi_{h c}, i \mathcal{L}_{\xi q}^{(1)}, i \mathcal{L}_{\xi q}^{(1)}, i \mathcal{L}_{\xi \xi}^{(0)}\right)=\bar{q} \bar{q} * \mathbf{J}_{L O} * \xi_{c} \xi_{c} \xi_{c},
\end{aligned}
$$

where the asterisks denote the convolutions with respect to collinear and soft fractions. The $T$-products which must be computed in SCET-I are shown explicitly, $\mathcal{L}_{\xi \xi}^{(0)}$ and $\mathcal{L}_{\xi q}^{(1)}$ denote the leading and subleading terms in the SCET-I Lagrangian, respectively. To the leading order accuracy we did not find any other combinations of the subleading terms which can describe the contributions with the soft spectators, in particular, the contribution with one soft spectator.

The matrix elements of the collinear operators in Eq.(19) yield twist-3 DAs (4) describing the initial and final protons, the soft fields $q$ are combined into SCF $\boldsymbol{S}$. Because the collinear operators in the both equations in (19) are the same, the functions $\mathbf{J}$ and $\mathbf{J}^{\prime}$ are also the same, therefore one needs to compute only one of it. Corresponding leading order diagrams are shown in Fig 2. The last two diagrams with

FIG. 2: Leading order SCET diagrams required for the calculation of jet functions. The inner dashed and curly lines denote hard-collinear quarks and gluons, external dashed lines correspond to collinear quarks, fermion lines with crosses denote soft quarks. Black square denotes the vertex of the SCET-I operator.

the three gluon vertex have vanishing color factors and therefore do not contribute. This is in agreement with the observation made in Ref. [14]. The soft-collinear vertices in the diagrams is obtained from the subleading SCET Lagrangian $\mathcal{L}_{\xi q}^{(1)}$, see details in [1]. Computing these diagrams we projected the Dirac indices according to our definitions of the DA (26) and SCF (15). We find that the contribution with 
$S_{T}$ corresponding to the tensor projection in eq. (16) has trivial coefficient function. This result is not an accident and can be explained by the angular momentum conservation (quite similar to discussion in [38]). Therefore we expect that corresponding hard kernel is trivial to all orders in $\alpha_{s}$.

In order to present the results for the jet functions let us rewrite Eq.(3) in the following form:

$$
F_{1}^{(s)}(Q)=C_{A}(Q)\left\{e_{u} f_{1}^{\mathrm{u}}(Q)+e_{d} f_{1}^{\mathrm{d}}(Q)\right\}
$$

where $e_{u, d}$ denote quark charges. The hard coefficient function $C_{A}(Q)$ in Eq.(20) includes all large logarithms so that SCET-I form factors $f_{1}^{\mathrm{u}, \mathrm{d}}$ depend only from the hard-collinear scale $Q \Lambda$ and defined as

$$
\left\langle p^{\prime}\left|\bar{\xi}_{h c}^{q} W^{\prime} \gamma_{\perp}^{\mu} W^{\dagger} \xi_{h c}^{q}\right| p\right\rangle_{\mathrm{SCET}}=\bar{N}\left(p^{\prime}\right) \frac{\not h h}{4} \gamma_{\perp}^{\mu} N(p) f_{1}^{q}\left(Q, \mu_{h c} \simeq Q \Lambda\right) \equiv f_{1}^{q}(Q),
$$

where the index $q$ describe the flavor of the hard-collinear field (active quark). Computing the diagrams in Fig 2 we obtained analytical expressions for the leading order jet functions. Our results can be presented in the following way

$$
\begin{aligned}
& f_{1}^{\mathrm{u}}(Q)=I_{A}^{\mathrm{u}}\left(\omega_{1}, \omega_{2}\right) * S_{A}^{\mathrm{ud}}\left(\omega_{1}, \omega_{2}, \nu_{1}, \nu_{2}\right) * I_{A}^{\mathrm{u}}\left(\nu_{1}, \nu_{2}\right)-I_{V}^{\mathrm{u}}\left(\omega_{1}, \omega_{2}\right) * S_{V}^{\mathrm{ud}}\left(\omega_{1}, \omega_{2}, \nu_{1}, \nu_{2}\right) * I_{V}^{\mathrm{u}}\left(\nu_{1}, \nu_{2}\right), \\
& f_{1}^{\mathrm{d}}(Q)=I_{A}^{\mathrm{d}}\left(\omega_{1}, \omega_{2}\right) * S_{A}^{\mathrm{uu}}\left(\omega_{1}, \omega_{1}, \nu_{1}, \nu_{2}\right) * I_{A}^{\mathrm{d}}\left(\nu_{1}, \nu_{2}\right)-I_{V}^{\mathrm{d}}\left(\omega_{1}, \omega_{2}\right) * S_{V}^{\mathrm{uu}}\left(\omega_{1}, \omega_{2}, \nu_{1}, \nu_{2}\right) * I_{V}^{\mathrm{d}}\left(\nu_{1}, \nu_{2}\right),
\end{aligned}
$$

where asterisk denotes the convolution integral with respect to the soft fractions, for instance

$$
I_{A}^{\mathrm{d}}\left(\omega_{1}, \omega_{2}\right) * S_{A}^{\mathrm{uu}}\left(\omega_{1}, \omega_{1}, \nu_{1}, \nu_{2}\right)=\int_{0}^{\infty} d \omega_{1} d \omega_{2} I_{A}^{\mathrm{d}}\left(\omega_{1}, \omega_{2}\right) S_{A}^{\mathrm{uu}}\left(\omega_{1}, \omega_{1}, \nu_{1}, \nu_{2}\right) .
$$

The proton DAs and integrations over the collinear fractions enter into the functions $I_{V, A}^{\mathrm{u}, \mathrm{d}}$ in Eqs. (22]23). These functions read:

$$
\begin{aligned}
& I_{V}^{\mathrm{u}}\left(\omega_{1}, \omega_{2}\right)=(V-A-2 T)\left(y_{1}, y_{2}, y_{3}\right) * J_{a}^{\mathrm{u}}\left(y_{1}, y_{2}, y_{3}, \omega_{1}, \omega_{2}\right) \\
&+(A-V-2 T)\left(y_{1}, y_{2}, y_{3}\right) * J_{b}^{\mathrm{u}}\left(y_{1}, y_{2}, y_{3}, \omega_{1}, \omega_{2}\right), \\
& I_{A}^{\mathrm{u}}\left(\omega_{1}, \omega_{2}\right)=(V-A-2 T)\left(y_{1}, y_{2}, y_{3}\right) * J_{b}^{\mathrm{u}}\left(y_{1}, y_{2}, y_{3}, \omega_{1}, \omega_{2}\right) \\
&+(A-V-2 T)\left(y_{1}, y_{2}, y_{3}\right) * J_{a}^{\mathrm{u}}\left(y_{1}, y_{2}, y_{3}, \omega_{1}, \omega_{2}\right), \\
& I_{V}^{\mathrm{d}}\left(\omega_{1}, \omega_{2}\right)=V\left(y_{1}, y_{2}, y_{3}\right) * J_{a}^{\mathrm{d}}\left(y_{1}, y_{2}, y_{3}, \omega_{1}, \omega_{2}\right)-A\left(y_{1}, y_{2}, y_{3}\right) * J_{b}^{\mathrm{d}}\left(y_{1}, y_{2}, y_{3}, \omega_{1}, \omega_{2}\right), \\
& I_{A}^{\mathrm{d}}\left(\omega_{1}, \omega_{2}\right)=-A\left(y_{1}, y_{2}, y_{3}\right) * J_{a}^{\mathrm{d}}\left(y_{1}, y_{2}, y_{3}, \omega_{1}, \omega_{2}\right),
\end{aligned}
$$

where the asterisk again denotes the convolution integral over collinear fractions:

$$
V\left(y_{1}, y_{2}, y_{3}\right) * J^{\mathrm{d}}\left(y_{1}, y_{2}, y_{3}, \ldots\right)=\int D y_{i} V\left(y_{1}, y_{2}, y_{3}\right) J^{\mathrm{d}}\left(y_{1}, y_{2}, y_{3}, \ldots\right) .
$$

The leading order hard-collinear jet-functions $J_{a, b}^{\mathrm{u}, \mathrm{d}}$ in Eqs.(25)(28) $\operatorname{read}\left(\bar{y}_{i}=1-y_{i}\right)$ :

$$
\begin{aligned}
& J_{a}^{\mathrm{u}}\left(y_{1}, y_{2}, y_{3} ; \omega_{1}, \omega_{2}\right)=\alpha_{s}^{2}\left(\mu_{h c}\right) \frac{8 \pi^{2}}{27} \frac{1}{\left(\omega_{1}+\omega_{2}\right) \omega_{1} \omega_{2}} \frac{1}{y_{2} y_{3}}\left(\frac{1}{\bar{y}_{2}}+\frac{1}{\bar{y}_{3}}-\frac{4\left(\omega_{1} y_{2}+\omega_{2} y_{3}\right)}{\bar{y}_{1}^{2}\left(\omega_{1}+\omega_{2}\right)}\right), \\
& J_{b}^{\mathrm{u}}\left(y_{1}, y_{2}, y_{3} ; \omega_{1}, \omega_{2}\right)=\alpha_{s}^{2}\left(\mu_{h c}\right) \frac{8 \pi^{2}}{27} \frac{1}{\left(\omega_{1}+\omega_{2}\right) \omega_{1} \omega_{2}} \frac{1}{\bar{y}_{2} \bar{y}_{3}}\left(\frac{1}{y_{3}}-\frac{1}{y_{2}}\right),
\end{aligned}
$$

and

$$
\begin{aligned}
& J_{a}^{\mathrm{d}}\left(y_{1}, y_{2}, y_{3} ; \omega_{1}, \omega_{2}\right)=\alpha_{s}^{2}\left(\mu_{h c}\right) \frac{8 \pi^{2}}{27} \frac{1}{\left(\omega_{1}+\omega_{2}\right) \omega_{1} \omega_{2}} \frac{1}{y_{1} y_{2}}\left(\frac{1}{\bar{y}_{1}}+\frac{1}{\bar{y}_{2}}-\frac{4\left(\omega_{1} y_{1}+\omega_{2} y_{2}\right)}{\bar{y}_{3}^{2}\left(\omega_{1}+\omega_{2}\right)}\right), \\
& J_{b}^{\mathrm{d}}\left(y_{1}, y_{2}, y_{3} ; \omega_{1}, \omega_{2}\right)=\alpha_{s}^{2}\left(\mu_{h c}\right) \frac{8 \pi^{2}}{27} \frac{1}{\left(\omega_{1}+\omega_{2}\right) \omega_{1} \omega_{2}} \frac{1}{\bar{y}_{1} \bar{y}_{2}}\left(\frac{1}{y_{2}}-\frac{1}{y_{1}}\right) .
\end{aligned}
$$


The argument of the QCD running coupling is defined by the hard-collinear scale: $\mu_{h c} \sim Q \Lambda$. From results (22) -(23) one can deduce that the convolution integrals in (25)-(28) with the proton DAs vanishing at the boundary as in Eq.(11) are well defined. Therefore one can assume that in the absence of other dominant regions, the convolution integrals with respect to soft fractions in (22)-(23) should also be finite. One can expect that the SCFs are concentrated in the region where the soft fractions is of order $\Lambda$ and fall quickly in the region where the soft fractions are much larger than $\Lambda$.

However the assumption of convergence is not correct. Using the perturbative expression (17) instead of nonperturbative function one can easily obtain, that any convolution integral with respect to the soft fractions is logarithmically divergent and proportional to $\sim \ln \left[\mu_{U V} / \mu_{I R}\right]$ where scale $\mu_{U V}$ and $\mu_{I R}$ represent the UV and IR cut-off respectively. This is exactly the logarithmic contribution which was found in [13] and later studied in [14] and called as "nonrenormalization group type logarithmic contribution".

Using the leading order expressions given in Eqs.(30)-(33) one can show that the similar situation also takes place for the nonperturbative SCFs. Recall that the soft fractions $\omega_{i}$ and $\nu_{i}$ can be associated with plus and minus projections of the soft momenta describing soft spectators. Therefore the boost invariance implies that SCF depends on the products $\omega_{i} \nu_{j}$ :

$$
S_{X}\left(\omega_{1}, \omega_{2}, \nu_{1}, \nu_{2}\right) \equiv S_{X}\left(\omega_{1} \nu_{1}, \omega_{1} \nu_{2}, \omega_{2} \nu_{2}, \omega_{2} \nu_{1}\right),
$$

Using this observation one can easily obtain that the convolution integrals of jet-functions and SCF in Eqs.(22)-(23) are divergent. Consider, as example, the integral from the Eq.(22):

$$
J=I_{A}^{\mathrm{u}}\left(\omega_{1}, \omega_{2}\right) * S_{A}^{\mathrm{ud}}\left(\omega_{1} \nu_{1}, \omega_{1} \nu_{2}, \omega_{2} \nu_{1}, \omega_{2} \nu_{2}\right) * I_{A}^{\mathrm{u}}\left(\nu_{1}, \nu_{2}\right),
$$

Using substitutions

$$
\nu_{1}=\nu_{1}^{\prime} / \omega_{1}, \nu_{2}=\nu_{2}^{\prime} / \omega_{1}, \omega_{2}=\omega_{2}^{\prime} \omega_{1}
$$

one obtains

$$
J=\int_{0}^{\infty} \frac{d \omega_{1}}{\omega_{1}} \int_{0}^{\infty} d \omega_{2}^{\prime} \int_{0}^{\infty} d \nu_{1}^{\prime} \int_{0}^{\infty} d \nu_{2}^{\prime} I_{A}^{\mathrm{u}}\left(\omega_{1}, \omega_{2}^{\prime} \omega_{1}\right) I_{A}^{\mathrm{u}}\left(\nu_{1}^{\prime} / \omega_{1}, \nu_{2}^{\prime} / \omega_{1}\right) S_{A}^{\mathrm{ud}}\left(\nu_{1}^{\prime}, \nu_{2}^{\prime}, \omega_{2}^{\prime} \nu_{1}^{\prime}, \omega_{2}^{\prime} \nu_{2}^{\prime}\right) .
$$

Using homogeneity of the leading order jet functions in Eqs.(30)-(33) yields

$$
I_{A}^{\mathrm{u}}\left(\omega_{1}, \omega_{2}^{\prime} \omega_{1}\right) I_{A}^{\mathrm{u}}\left(\nu_{1}^{\prime} / \omega_{1}, \nu_{2}^{\prime} / \omega_{1}\right)=I_{A}^{\mathrm{u}}\left(1, \omega_{2}^{\prime}\right) I_{A}^{\mathrm{u}}\left(\nu_{1}^{\prime}, \nu_{2}^{\prime}\right)
$$

and one obtains

$$
J=\int_{0}^{\infty} \frac{d \omega_{1}}{\omega_{1}} \int_{0}^{\infty} d \omega_{2}^{\prime} \int_{0}^{\infty} d \nu_{1}^{\prime} \int_{0}^{\infty} d \nu_{2}^{\prime} I_{A}^{\mathrm{u}}\left(1, \omega_{2}^{\prime}\right) I_{A}^{\mathrm{u}}\left(\nu_{1}^{\prime}, \nu_{2}^{\prime}\right) S_{A}^{\mathrm{ud}}\left(\nu_{1}^{\prime}, \nu_{2}^{\prime}, \omega_{2}^{\prime} \nu_{1}^{\prime}, \omega_{2}^{\prime} \nu_{2}^{\prime}\right) .
$$

The first integral in the last Eq.(39) is divergent $\int_{0}^{\infty} \frac{d \omega_{1}}{\omega_{1}} \sim \ln \left[\mu_{U V} / \mu_{I R}\right]$. The similar arguments can be used for the all contributions in Eqs.(22)-(23). Then, for instance, for the $u$-quark form factor in Eq.(22) one obtains

$$
\begin{aligned}
& f_{1}^{\mathrm{u}}(Q)=\int_{0}^{\infty} \frac{d \omega_{1}}{\omega_{1}} \int_{0}^{\infty} d \omega_{2}^{\prime} \int_{0}^{\infty} d \nu_{1}^{\prime} \int_{0}^{\infty} d \nu_{2}^{\prime}\left\{I_{A}^{\mathrm{u}}\left(1, \omega_{2}^{\prime}\right) I_{A}^{\mathrm{u}}\left(\nu_{1}^{\prime}, \nu_{2}^{\prime}\right) S_{A}^{\mathrm{ud}}\left(\nu_{1}^{\prime}, \nu_{2}^{\prime}, \omega_{2}^{\prime} \nu_{1}^{\prime}, \omega_{2}^{\prime} \nu_{2}^{\prime}\right)\right. \\
& \left.-I_{V}^{\mathrm{u}}\left(1, \omega_{2}^{\prime}\right) I_{V}^{\mathrm{u}}\left(\nu_{1}^{\prime}, \nu_{2}^{\prime}\right) S_{V}^{\mathrm{ud}}\left(\nu_{1}^{\prime}, \nu_{2}^{\prime}, \omega_{2}^{\prime} \nu_{1}^{\prime}, \omega_{2}^{\prime} \nu_{2}^{\prime}\right)\right\},
\end{aligned}
$$

The the coefficient in front of divergent factor depends on the different combinations of different nonperturbative functions and we do not see any arguments which allows one to conclude that this coefficient may vanish. Hence we can conclude that the soft convolution integrals in the soft spectator contribution are divergent and this singularity is independent on the particular properties of SCF. The same observation can also be done for the $d$-quark form factor in $\mathrm{Eq}(23)$.

In conclusion, we have shown by the explicit leading order calculation of the jet functions that the soft spectator contribution is not well defined. The convolution integrals with respect to the soft fractions are divergent and these, so-called, end-point singularities do not cancel in the sum of all diagrams.

Is this divergency a signal that the factorization scheme suggested in Eqs.(1]-3) is incomplete? The form of the divergent contribution allows one to assume that dimensionless integral $\int_{0}^{\infty} \frac{d \omega_{1}}{\omega_{1}}$ indicates that the 
soft region overlaps with the collinear one as it happens, for instance, in the classical case of Sudakov form factor [33]. Then we may also expect that contributions associated with the collinear regions overlap with the soft region. If such situation takes place then we must observe IR-divergencies in the collinear convolution integrals which describe the hard rescattering term and also referred as end-point divergencies.

However, the corresponding collinear integrals computed with the existing models of DA, like one in Eq.(10), are well defined. At first glance such situation may look as contradictory, however it is not like that. In order to explain this observation one has to take into account following arguments.

First, the end-point behavior of the DA models Eq.(10) is closely associated with the end-point behavior of the QCD evolution. In particular, the asymptotic DA is the eigenfunction of the evolution kernel with the smallest eigenvalue which is the anomalous dimension of the corresponding three quark operator.

The second observation is given by consideration of the soft correlation function in perturbation theory. We obtained that the first nontrivial contribution is associated with the masses of the soft spectators, see Eq. (17). On the other hand for the calculation of the evolution kernel the mass of the quarks is not needed and the chiral symmetry in perturbation theory is not broken. The terms proportional to the masses in such calculation are finite and as a rule neglected. But for the calculation of the soft spectator contribution in perturbation theory the presence of the soft spectator quark masses is necessary, see details in Appendix B. The introduction of mass for the soft spectator quarks in the perturbative calculations is directly related with the chiral symmetry breaking in pQCD. Then we may conclude that the mechanism of the violation of the factorization in our case is closely associated with chiral symmetry breaking and therefore it can not be observed if this symmetry is preserved. Such scenario may explain why we do not observe any end-point singularities in the collinear integrals computed with the models like one in Eq. (10) for which the end-point behavior is strongly motivated by QCD evolution.

In order to verify this picture and to clarify the mechanism of the collinear and soft overlap in QCD we suggest to perform the analysis of the factorization in the perturbative sector with massive quarks. In this case the mass plays the role of the soft scale and we can unambiguously define the all soft matrix elements in the perturbation theory. In he collinear sectors this allows us to compute not only the logarithmic contributions associated with the evolution of DA but also the finite contributions which can appear due to quark mass.

In the next section we present investigation of the factorization properties in pQCD using as example the 2-loop diagram which has both contributions associated with the soft and collinear sectors.

\section{SOFT-COLLINEAR OVERLAP AND SEPARATION OF THE HARD AND SOFT SPECTATOR SCATTERING CONTRIBUTIONS}

\section{A. Overlap of the soft and collinear regions}

In order to perform the required analysis we consider the diagram shown in Fig 3 . This diagram was discussed in [1] in order to show nontrivial contribution arising from the soft region. But we did not investigate the possibility of the overlap of the soft region with other dominant regions. We consider this more accurately now because it will help us to solve the problem with the soft divergent integral and understand the factorization for $F_{1}$. The contribution of this diagram into the nucleon FF $F_{1}$ can be

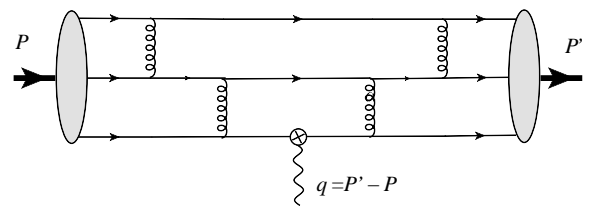

FIG. 3: Two-loop diagram which obtain contributions from the hard, collinear and soft regions.

written as

$$
\bar{N}^{\prime} \gamma_{\perp}^{\mu} N F_{1}[D]=\int D x_{i} \int D y_{i} \Psi_{\boldsymbol{\alpha}}\left(y_{i}\right)\left[D^{\mu_{\perp}}\left(x_{i}, y_{i}\right)\right]_{\boldsymbol{\alpha} \boldsymbol{\beta}} \boldsymbol{\Psi}_{\boldsymbol{\beta}}\left(x_{i}\right)
$$


where DA $\boldsymbol{\Psi}$ is associated with the blobs in Fig 3 , In order to simplify our consideration we use simple observation which follow from Eq.(6):

$$
\boldsymbol{\Psi}\left(y_{i}\right) \not=0, \not h \boldsymbol{\Psi}\left(x_{i}\right)=0 .
$$

This allows us in the intermediate calculations to substitute instead of full DA $\Psi$ the large components of collinear spinors:

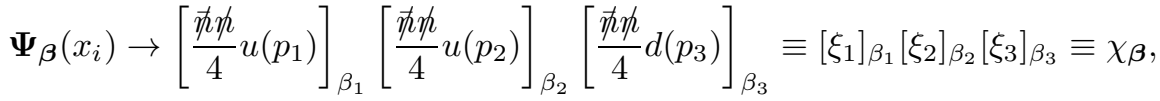

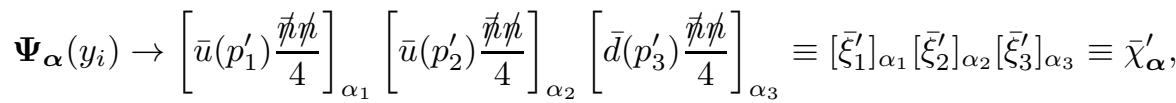

For simplicity we always assume that the bottom fermion line in the diagram corresponds to $d$-quark. We always assume that color indices are properly contracted and don't show them explicitly. In order to simplify our consideration we will not contract the Dirac indices and compute corresponding traces. It is also clear that relation of the perturbative expressions with the corresponding realistic amplitudes with nonperturbative DAs can be obtained by re-substitution $\chi \rightarrow \Psi$.

Therefore the contribution of the diagram in Fig 3 can be associated with the perturbative diagram in Fig 4 and corresponding analytical expression reads:

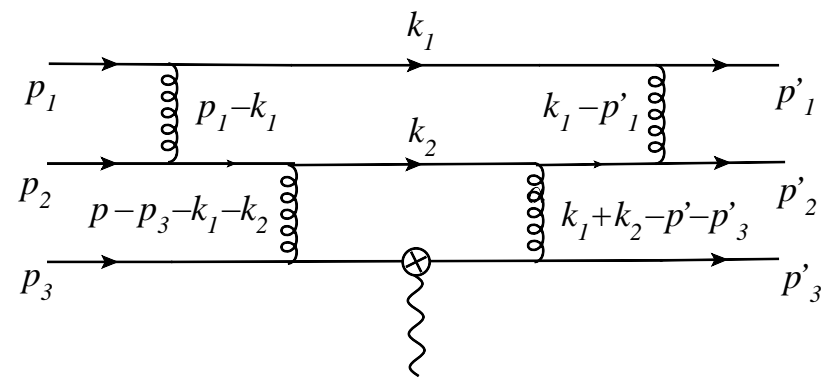

FIG. 4: Two-loop diagram with momenta flow.

$$
\begin{aligned}
D^{\mu} & =\mathcal{C} \int d^{4} k_{1} d^{4} k_{2} \frac{1}{\left[k_{1}^{2}-m^{2}\right]\left[k_{2}^{2}-m^{2}\right]} \\
& \times \frac{\bar{\xi}_{1}^{\prime} \gamma^{i}\left(\hat{k}_{1}+m\right) \gamma^{j} \xi_{1} \bar{\xi}_{2}^{\prime} \gamma^{i}\left(\hat{p}^{\prime}-\hat{p}_{3}^{\prime}-\hat{k}_{1}+m\right) \gamma^{\alpha}\left(\hat{k}_{2}+m\right) \gamma^{\beta}\left(\hat{p}-\hat{p}_{3}-\hat{k}_{1}+m\right) \gamma^{j} \xi_{2}}{\left[\left(p-p_{3}-k_{1}\right)^{2}-m^{2}\right]\left[\left(p^{\prime}-p_{3}^{\prime}-k_{1}\right)^{2}-m^{2}\right]\left(k_{1}+k_{2}-p^{\prime}+p_{3}^{\prime}\right)^{2}\left(p-p_{3}-k_{1}-k_{2}\right)^{2}} \\
& \times \frac{\bar{\xi}_{3}^{\prime} \gamma^{\alpha}\left(\hat{p}^{\prime}-\hat{k}_{1}-\hat{k}_{2}+m\right) \gamma^{\mu}\left(\hat{p}-\hat{k}_{1}-\hat{k}_{2}+m\right) \gamma^{\beta} \xi_{3}}{\left[\left(p^{\prime}-k_{1}-k_{2}\right)^{2}-m^{2}\right]\left[\left(p-k_{1}-k_{2}\right)^{2}-m^{2}\right]\left(p_{1}-k_{1}\right)^{2}\left(k_{1}-p_{1}^{\prime}\right)^{2}}
\end{aligned}
$$

The quark mass $m$ is used as a soft scale in order to regularize IR-divergencies and to describe the soft contribution, see Eq.(17). The factor $\mathcal{C}$ accumulates color structures and others factors according to Feynman rules.

Our task is to clarify the relation between the collinear and soft regions performing the interpretation of $D^{\mu}$ in terms of different contributions according to factorization formulas (2) and (3). If the overlap of these sectors is not possible then we may be find the missing elements in the factorization description. Notice that diagram in Fig 4 does not have UV-divergent subgraphs and therefore we do not need to consider renormalization of QCD parameters like quark mass and running coupling. Taking into account the logarithmic structure of the hard spectator term $F_{1}^{(h)}$ we may expect that after 2-loop integration the answer can be schematically written as

$$
D^{\mu_{\perp}}=\frac{1}{Q^{6}}\left[A^{\mu_{\perp}} \ln ^{2} Q^{2} / m^{2}+B^{\mu_{\perp}} \ln Q^{2} / m^{2}+C^{\mu_{\perp}}\right]+\mathcal{O}\left(1 / Q^{7}\right) .
$$


where the leading power $Q^{-6}$ is obtained from the dimension reasons. In the absence of the divergent soft contribution we could expect that amplitudes $A$ and $B$ can be interpreted in terms of convolutions of the LO and NLO hard coefficient functions with the two- and one-loop evolution kernels, respectively. However, the divergency of the soft convolution integral in (3) allows us to suggest that there is a large logarithm of $Q$ which has a different interpretation.

The exact calculation of the coefficients $A, B, C$ in Eq.(46) is a difficult task involving 2-loop massive integrals and we are not going to proceed in this way. We will focus our attention on the possible overlap of the soft and collinear contributions which can most probably provide the solution of the problem. The idea is to use the strategy of regions [39, 40] technique which allows one to find and interpret the contributions originated from the different sectors in (45). This formalism also allows one to establish wether the collinear contributions overlap with the soft sectors.

Let us begin our discussion from the soft region where

$$
k_{1 \mu} \sim k_{2 \mu} \sim \Lambda,
$$

Corresponding contribution can be represented as [1]:

$$
\begin{aligned}
& D_{s}^{\mu_{\perp}}=\mathcal{C} \bar{\chi}_{\boldsymbol{\beta}}\left[\left(\gamma_{\perp}^{\mu}\right)_{\beta_{3} \alpha_{3}}\right]_{C_{A}} \chi_{\boldsymbol{\alpha}} \int d k_{1,2}^{+}\left[\frac{\left[\gamma^{i}\right]_{\beta_{1} \alpha_{1}^{\prime}}\left[\gamma^{i}\right]_{\beta_{2} \alpha_{2}^{\prime}}}{y_{1} \bar{y}_{3}^{2}\left[-Q\left(k_{1}^{+}+k_{2}^{+}\right)\right]^{2}\left[-Q k_{1}^{+}\right]}\right]_{J^{\prime}} \\
& \int d k_{1,2}^{-}\left[\frac{\left[\gamma^{j}\right]_{\beta_{1}^{\prime} \alpha_{1}}\left[\gamma^{j}\right]_{\beta_{2}^{\prime} \alpha_{2}}}{x_{1} \bar{x}_{3}^{2}\left[-Q\left(k_{1}^{-}+k_{2}^{-}\right)\right]^{2}\left[-Q k_{1}^{-}\right]}\right]_{J}\left[\int d k_{12 \perp} \frac{\left(\hat{k}_{1}+m\right)_{\alpha_{1}^{\prime} \beta_{1}^{\prime}}\left(\hat{k}_{2}+m\right)_{\alpha_{2}^{\prime} \beta_{2}^{\prime}}}{\left[k_{1}^{2}-m^{2}\right]\left[k_{2}^{2}-m^{2}\right]}\right]_{S} .
\end{aligned}
$$

The subscripts $C_{A}, J, S$ are associated with the appropriate contributions in Eq.(3) so that expressions in the brackets can be associated with these quantities in pQCD.

Computing the transverse integrals inside the brackets associated with SCF $S$ one obtains the answer similar to one given in Eq. (17) which is proportional to the mass $m^{2}$. Redefining the light-cone variables $k_{1,2}^{ \pm}$in the convolution integrals one can eliminate the mass $m$ from the consideration. But such redefinition performed in the divergent integrals may lead to a mistake. We will see that calculation of the regularized soft integrals leads to a logarithmic dependence on the mass $m$.

We find that the contributions of the remaining regions in $D^{\mu}$ can be associated only with the hard rescattering term $F_{1}^{(h)}$ (2). Corresponding contributions are described by the different combinations of the collinear and hard regions. For brevity we skip the discussion of the all dominant regions and pass directly to those which are relevant to our consideration. These regions can be described as collinear regions, where both spectator momenta $k_{1,2}$ collinear either to initial $p$ or to final $p^{\prime}$ momentum. Such scenario looks understandable taking into account that we are looking for overlap with two soft spectators. Because of the symmetry between in and out states in Eq.(45) it is enough to consider only one of them. Let us choose

$$
k_{1} \sim k_{2} \sim p^{\prime}: k_{i}^{-} \sim Q, k_{+} \sim \Lambda^{2} / Q, k_{\perp} \sim \Lambda .
$$

Then one obtains that corresponding contribution has the leading power suppression $Q^{-6}$ as in Eq.(46) and can be written as a convolution of hard $[\ldots]_{\mathrm{H}}$ and collinear $\mathcal{V}$ parts (see details in Appendix $\mathrm{C}$ ):

$$
D_{c p^{\prime}}^{\mu_{\perp}} \simeq \mathcal{C} \bar{\chi}_{\boldsymbol{\beta}}^{\prime} \int d k_{1}^{-} d k_{2}^{-} \mathcal{V}_{\boldsymbol{\beta} \boldsymbol{\beta}^{\prime}}\left(k_{i}^{-}\right)\left[\frac{\left[\gamma^{j}\right]_{\beta_{1}^{\prime} \alpha_{1}}\left[\gamma^{j}\right]_{\beta_{2}^{\prime} \alpha_{2}}\left[\gamma_{\perp}^{\mu}\right]_{\beta_{3}^{\prime} \alpha_{3}}}{\bar{x}_{3}^{2} x_{1}\left[-Q\left(k_{1}^{-}+k_{2}^{-}\right)\right]^{2}\left[-Q k_{1}^{-}\right]}\right]_{\mathrm{H}} \chi_{\boldsymbol{\alpha}}
$$

where

$$
\begin{aligned}
\mathcal{V}\left(k_{i}^{-}\right)= & \frac{1}{2} \int d k_{1}^{+} d k_{2}^{+} d k_{1 \perp} d k_{2 \perp} \frac{\left\{\gamma^{i}\left(\hat{k}_{1}+m\right)\right\}_{\beta_{1} \beta_{1}^{\prime}}}{\left[k_{2}^{2}-m^{2}\right]\left[k_{1}^{2}-m^{2}\right]} \\
& \times \frac{\left\{\gamma^{i}\left(\hat{p}^{\prime}-\hat{p}_{3}^{\prime}-\hat{k}_{1}+m\right) \gamma^{\alpha}\left(\hat{k}_{2}+m\right)\right\}_{\beta_{2} \beta_{2}^{\prime}}\left\{\gamma^{\alpha}\left(\hat{p}^{\prime}-\hat{k}_{1}-\hat{k}_{2}+m\right)\right\}_{\beta_{3} \beta_{3}^{\prime}}}{\left[\left(p^{\prime}-k_{1}-k_{2}\right)^{2}-m^{2}\right]\left[\left(p^{\prime}-p_{3}^{\prime}-k_{1}\right)^{2}-m^{2}\right]\left(k_{1}+k_{2}-p^{\prime}+p_{3}^{\prime}\right)^{2}\left(k_{1}-p_{1}^{\prime}\right)^{2}} .
\end{aligned}
$$




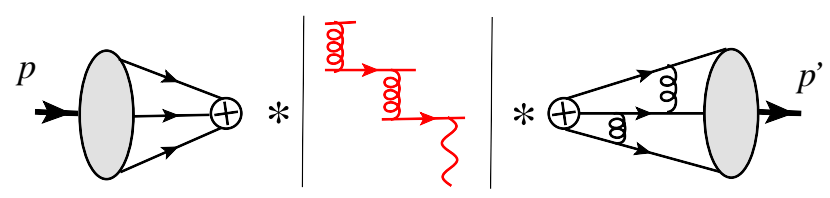

FIG. 5: Graphical interpretation of the $D_{c p^{\prime}}^{\mu_{\perp}}$ given by Eq. (50). The hard subdiagram with the two gluon exchange is separated by vertical line and shown by red lines in the color picture. The asterisk denotes the integral convolution wit respect to collinear fractions.

The interpretation of this contribution is given in Fig 5

The collinear part $\mathcal{V}$ can be clearly associated with the two-loop contribution to the evolution kernel of the DA. Recall, that light-cone components $k_{ \pm}$scale according to (49). Computing the integrals over $k_{1,2}^{+}$in Eq.(51) we obtain that the integration region for the minus components are restricted $0<k_{i}^{-}<$ $Q$. Therefore these variables can be rescaled to the dimensionless quantities which, as a rule, used as collinear fractions. The integrals over the transverse momenta in (51) are UV-divergent and these are the logarithmical divergencies associated with the evolution of DA.

In order to see the overlap of the soft and collinear regions we consider the soft limit (47) in the collinear contribution $D_{c p^{\prime}}^{\mu_{\perp}}$ in Eq. (50). In this case one finds that the collinear-soft limit reproduces the soft contribution in Eq.(48): $\left(D_{c p^{\prime}}^{\mu_{\perp}}\right)_{s}=D_{s}^{\mu_{\perp}}$. This allows us to conclude that collinear and soft regions in this case overlap. As a consequence, this also allows us to expect that the convolution integrals with respect to $k_{-}$in (50) are singular at the end-point region. We will see this explicitly computing the collinear integral (50) in Sec IIB.

Let us emphasize one important point. Analysis of the soft contribution in Eq.(48) shows that the terms with momenta $\hat{k}_{1,2}$ in the numerator of the integrand vanishes so that

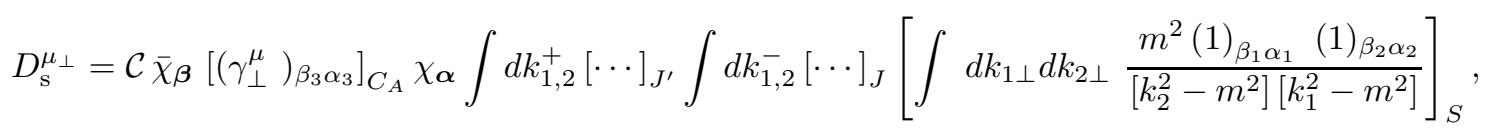

This can be easily seen if one uses the Sudakov decomposition for the soft momenta $k_{1,2}$ in the soft part in Eq.(48). Then the terms with the longitudinal components $k_{i}^{ \pm} \gamma_{\mp}$ vanish due Dirac projections on the leading twist DAs (42). The part with the transverse projections $\not 1_{1} \perp k_{2} \perp$ vanishes due to the rotation invariance in transverse subspace (see also Appendix B). Therefore we obtain that only $\sim m^{2}$ part can provide nontrivial contribution. The specific role of the mass squared term in discussion of the soft spectator scattering was also noted in 12 .

From this observation we can conclude that the appropriate part of the collinear contribution $D_{c p^{\prime}}^{\mu_{\perp}}$ in Eq.(50) describing the soft collinear overlap is UV-finite. Thus we obtain that the intersection of the soft collinear regions in the given example does not involve to the UV-divergencies of the collinear operator which is associated with the QCD evolution of nucleon DA.

Taking into account this fact we can conclude that the convolution integrals of the hard coefficient function $\mathbf{H}$ in the factorization formula (2) with 1- and 2-loop evolution kernels $\mathcal{V}_{1,2}$ or with the models of DA with the vanishing end-point behavior (11) (for instance, like (10)) are free from the end-point singularities. But the convolution of LO hard coefficient function $H$ in Eq.(150) with the finite $m^{2}$-term originating from the collinear subdiagram $\mathcal{V}$ is singular. Below we will demonstrate that this singularity provides a logarithmic contribution which in the sum with the UV-logarithm from the soft term (48) yields a contribution proportional to $\ln Q / m$.

Such situation turns out to be different from the many well known cases when the end-point singularities appear after the inspection of the end-point behavior of the evolution kernel, or equivalently, asymptotic DA. Such situation takes place, for instance, in the case of Pauli FF $F_{2}$. Usually in these cases one deals with the higher twist DAs. These DAs describe the higher (excited) components of the hadronic wave function and, as a rule, can be associated with the orbital motion of the partons.

But in case of $F_{1}$ the situation is different. We always consider only the leading twist projections and therefore we do not involve any higher twist DAs. Therefore the mechanism of the factorization breaking in this case is different. Taking into account the role of the soft quark masses we can conclude that in this case violation of the collinear factorization can be associated with the chiral symmetry breaking. 
Therefore we can conclude that combination of two possibilities: soft spectator scattering together with chiral symmetry breaking provides the specific mechanism in pQCD which leads to the soft collinear overlap and breaks the collinear factorization. This scenario also explains why we do not see any problem with the collinear convolution integrals when we use DAs associated with the evolution kernels. The QCD evolution is not sensitive to the chiral symmetry breaking: the evolution kernels can be computed with the massless quarks. But how the chiral symmetry breaking can affect the DA in order to provide the singular convolution? In order to answer this question we have to find the interpretation of the UV-finite contribution arising in $\mathcal{V}$ in Eq. (50) .

Let us consider the perturbative analog of the proton DA, i.e. as we would substitute three collinear quark states instead of protons. In such case the 3-quark DA is defined as pQCD matrix element and its perturbative expansion schematically read ${ }^{1}$ :

$$
\mathbf{\Psi}_{\mathrm{PT}}\left(x_{i}, \mu_{F}\right)=\Psi_{0}\left(x_{i}\right)+\frac{\alpha_{s}\left(\mu_{R}\right)}{\pi} \Psi_{1}\left(x_{i}, \mu_{F}\right)+\left(\frac{\alpha_{s}\left(\mu_{R}\right)}{\pi}\right)^{2} \Psi_{2}\left(x_{i}, \mu_{F}\right)+\ldots .
$$

where the higher order coefficients $\Psi_{i}\left(x_{i}, \mu_{F}\right), i>0$ depend logarithmically on the renormalization (or factorization) scale $\mu_{F}$. Schematically this can be represented as:

$$
\begin{aligned}
& \Psi_{1}\left(x_{i}, \mu_{F}\right)=\ln \mu_{F}^{2} / m^{2} \mathcal{V}_{1} * \Psi_{0}+\Psi_{10}\left(x_{i}\right) \\
& \Psi_{2}\left(x_{i}, \mu_{F}\right)=\ln ^{2} \mu_{F}^{2} / m^{2} \mathcal{V}_{1} * \mathcal{V}_{1} * \Psi_{0}+\ln \mu_{F}^{2} / m^{2} \mathcal{V}_{2} * \Psi_{0}+\Psi_{20}\left(x_{i}\right)+\ldots
\end{aligned}
$$

where asterisk as usually denotes the collinear convolution integrals, $\mathcal{V}_{1,2}$ denote now 1 - or 2-loop evolution kernels and dots represent the other contributions associated with the renormalization of QCD coupling. If we put $\mu_{F}=m$ then all large logarithms vanish and we obtain:

$$
\boldsymbol{\Psi}_{\mathrm{PT}}\left(x_{i}, m\right)=\Psi_{0}\left(x_{i}\right)+\frac{\alpha_{s}(m)}{\pi} \Psi_{10}\left(x_{i}\right)+\left(\frac{\alpha_{s}(m)}{\pi}\right)^{2} \Psi_{20}\left(x_{i}\right)+\ldots .
$$

Obviously, Eq.(56) is understood as a perturbative expansion of the DA at the low energy scale $m \ll$ Q. Performing transition from perturbative consideration to physical FF one substitutes instead of perturbative DA the realistic one: $\boldsymbol{\Psi}_{\mathrm{PT}}\left(x_{i}, m\right) \rightarrow \boldsymbol{\Psi}\left(x_{i}, \mu_{0}\right)$, where it is natural to assume that $\mu_{0}$ is a certain soft scale of order $\Lambda$. Following these arguments we can perform the interpretation of the UV-finite term $\sim m^{2}$ in Eq.(51). This term provides the contribution to $\Psi_{20}\left(x_{i}\right)$ and therefore can be associated with the DA at low normalization $\mu_{0}$. If $\Psi_{20}\left(x_{i}\right)$ decreases more slowly at small values $x_{i} \rightarrow 0$ comparing to the asymptotic DA $\boldsymbol{\Psi}_{a s}\left(x_{i} \rightarrow 0\right) \sim x_{i} x_{j}$ then the corresponding collinear convolution integrals can be singular. Below we will demonstrate that in $\mathrm{pQCD}$ one obtains the linear behavior

$$
\Psi_{20}\left(x_{i} \rightarrow 0\right) \sim x_{i},
$$

assuming that the ratio of the small fractions $x_{i} / x_{j}$ is fixed. Let us emphasize again that such a behavior is strongly motivated by the presence of soft spectator contribution.

Concluding let us mention that similar analysis can be also carried out for other QCD diagrams which have both contributions associated with the hard and soft spectator scattering. We are not going to present it for all of them because it does not provide us with any new principal information, on the other hand such analysis is very lengthy and technically complicate. But in the next section we will continue to study the overlap of the collinear and soft sectors in the framework of pQCD and present the explicit results for the collinear and soft contributions discussed above.

\section{B. Calculation of the large logarithmic term arising from the overlap of collinear and soft regions}

Having established that for the soft-collinear overlap one faces the problem separating unambiguously the regions in the factorization formulas (2) and (3). This is quite a complicated problem, especially for

\footnotetext{
${ }^{1}$ For brevity we skip the Dirac and color indices
} 
the processes involving composite particles like hadrons. Therefore it is useful as a first step to consider more simple examples with similar features. We will continue the discussion of the diagram in Fig,4 and discuss the separation of the soft and collinear contributions in this particular case.

In order to compute the contributions of the soft (48) and collinear (50) regions explicitly we need a specific regularization for the convolution integrals over longitudinal momenta. Dimensional regularization (DR) can not be used in this situation because the soft contribution is given by scaleless integral and therefore equals to zero in this case. Therefore one has to introduce a different regularization in order to work with the collinear and soft integrals in (2) and (3). This is not only a technical problem. A careful prescription is required in order to avoid double counting computing the different contributions. These questions have been studied during last years in many publications in connection with the Sudakov form factor, see e.g. [33, 46, 47]. In [33] a systematic subtraction procedure has been suggested in order to separate contributions of collinear and soft modes in non-inclusive processes. The factorization in the context of SCET was discussed in [34] where the idea of the so-called zero-bin subtractions was invented. In our analysis we adopt this technique in order to formulate a prescription for the correct separation of the soft and collinear modes and compute required integrals.

Note that the soft contribution in Eq.(48) and the appropriate part of the collinear term in Eq.(50) do not overlap with the hard region. As a result these integrals can be considered as UV-finite (in a sense that integrals over transverse momenta are finite). This allows us to carry out all calculations using a specific regularization in four dimensions which may be the simplest solution in this case. Such regularization prescription must be formulated uniformly for the all collinear and soft divergent integrals. There is also a technical problem about applicability of the method of regions with this specific regularization in $D=4$. In order to fix these details we suggest to investigate a simple one-loop integral which is close to our situation and can be easily computed.

\section{Collinear and soft contributions in $D=4$ : one-loop case}

As example consider following integral:

$$
J=\int d k \frac{m^{2}}{\left[k^{2}-m^{2}\right]^{2}} \frac{1}{\left[k^{2}-2(p k)\right]} \frac{1}{\left[k^{2}-2\left(p^{\prime} k\right)\right]}=\frac{i \pi}{Q^{2}} \ln Q^{2} / m^{2}+O\left(m^{2} / Q^{2}\right) .
$$

where we assume that expressions in the square brackets are defined with the $+i \varepsilon$ prescription: $[X] \equiv$ $[X+i \varepsilon]$, and the momenta $p$ and $p^{\prime}$ are the same as used before. It is easily to see that this integral can be related to a well-known scalar vertex integral:

$$
J=m^{2} \frac{d}{d m^{2}} \int d k \frac{1}{\left[k^{2}-m^{2}\right]} \frac{1}{\left[k^{2}-2(p k)\right]} \frac{1}{\left[k^{2}-2\left(p^{\prime} k\right)\right]}
$$

The asymptote of the vertex integral is given by large Sudakov logarithm $\ln ^{2} Q^{2} / m^{2}$ but the mass differentiation reduces this structure to a simple logarithm. Analysis of the dominant regions yields:

$$
J_{\text {coll }} \sim J_{s} \sim \frac{1}{Q^{2}}, \quad J_{h} \sim \frac{m^{2}}{Q^{4}},
$$

where the subscripts "coll", "s" and "h" denotes collinear to $p$ or $p^{\prime}$, soft and hard regions respectively. The hard region is suppressed and the large logarithm is generated only from the overlap of collinear and soft regions. In order to obtain the leading order result (58) with the help of the method of regions we must introduce appropriate regularization. It is not difficult to see that DR can not help in this case.

Consider the regularization by small off-shell external momenta introducing the small transverse components. This yields:

$$
J_{\text {reg }}=\int d k \frac{m^{2}}{\left[k^{2}-m^{2}\right]^{2}} \frac{1}{\left[k^{2}-2(p k)-p_{\perp}^{2}\right]} \frac{1}{\left[k^{2}-2\left(p^{\prime} k\right)-p_{\perp}^{\prime 2}\right]}
$$

As usually, one may expect that the exact answer (58) can be reproduced by the sum of the contributions from the dominant regions:

$$
J \simeq J_{c p^{\prime}}+J_{c p}+J_{s}
$$


But in this case this not true and this can be easily seen from the explicit calculation. The contribution from the soft region reads:

$$
J_{s} \simeq \int d k \frac{m^{2}}{\left[k^{2}-m^{2}\right]^{2}} \frac{1}{\left[-p_{+} k_{-}-p_{\perp}^{2}\right]} \frac{1}{\left[-p_{-}^{\prime} k_{+}-p_{\perp}^{2}\right]}=\frac{1}{Q^{2}} \int d k \frac{m^{2}}{\left[k^{2}-m^{2}\right]^{2}} \frac{1}{\left[-k_{-}-\tau_{-}\right]} \frac{1}{\left[-k_{+}-\tau_{+}\right]}
$$

where we introduced $\tau_{-}=p_{\perp}^{2} / p_{+}$and $\tau_{+}=p_{\perp}^{\prime 2} / p_{-}^{\prime}$. Notice that in the absence of the regulators this soft integral is scaleless and has both UV and IR divergencies as the integral in Eq.(39). Performing integration over $k_{-}$by residues and taking the transverse integral one obtains

$$
J_{s}=\frac{i \pi}{Q^{2}} \int_{0}^{\infty} d k_{+} \frac{1}{\left[k_{+}+\tau_{+}\right]} \frac{1}{\left[1+k_{+} \tau_{-} / m^{2}\right]}=-\frac{i \pi}{Q^{2}} \frac{m^{2} \ln \left[\tau_{+} \tau_{-} / m^{2}\right]}{\tau_{+} \tau_{-}-m^{2}} \simeq-\frac{i \pi}{Q^{2}} \ln \left[\tau_{+} \tau_{-} / m^{2}\right] .
$$

From Eq. (64) it is clearly seen that the regulators $\tau_{ \pm}$serves as IR- and UV-regulators $\left(k_{+} \rightarrow 0\right.$ or $k_{+} \rightarrow \infty$ respectively). Passing to the last equation in (64) we neglected the regular in $\tau_{+} \tau_{-}$contributions assuming $\tau_{+} \tau_{-} \ll m^{2}$.

In collinear region $k \sim p^{\prime}$ one obtains

$$
\begin{aligned}
J_{c p^{\prime}} & =\int d k \frac{m^{2}}{\left[k^{2}-m^{2}\right]^{2}} \frac{1}{\left[-2(p k)-p_{\perp}^{2}\right]} \frac{1}{\left[\left(p^{\prime}-k\right)^{2}-p_{\perp}^{\prime 2}\right]} \\
& =\frac{1}{p_{+}} \int d k_{-} \frac{1}{\left[-k_{-}-\tau_{-}\right]} \frac{1}{2} \int d k_{\perp} d k_{+} \frac{m^{2}}{\left[k^{2}-m^{2}\right]^{2}} \frac{1}{\left[k^{2}-2 p_{-}^{\prime} k_{+}\right]} .
\end{aligned}
$$

We neglected the second regulator $\tau_{+}$in (66) because the corresponding integral is finite. Again, computing the integrals over $k_{+}$and $k_{\perp}$ we obtain

$$
J_{c p^{\prime}}=\frac{i \pi}{p_{+}} \int_{0}^{p_{-}^{\prime}} d k_{-} \frac{1}{\left[k_{-}+\tau_{-}\right]}=-\frac{i \pi}{Q^{2}} \ln \frac{\tau_{-}}{p_{-}^{\prime}} .
$$

Similarly one computes the second collinear integral $k \sim p$ :

$$
J_{c p}=-\frac{i \pi}{Q^{2}} \ln \frac{\tau_{+}}{p_{+}} .
$$

However the sum of the all terms (62) can not reproduce the exact answer (58). The reason is that collinear integrals $J_{c p^{\prime}}$ and $J_{c p}$ obtain contribution also from the soft region. Computing the soft limit in expression in (66)

$$
\left(J_{c p^{\prime}}\right)_{s}=\int d k \frac{m^{2}}{\left[k^{2}-m^{2}\right]^{2}} \frac{1}{\left[-2(p k)-p_{\perp}^{2}\right]} \frac{1}{\left[-2\left(p^{\prime} k\right)-p_{\perp}^{\prime 2}\right]}
$$

we reproduce expression for the soft integral in (63). Hence in order to compute the contribution of the collinear regions correctly one has to subtract from the expressions for collinear integrals $J_{c p^{\prime}}$ and $J_{c p}$ appropriate soft contributions [33] (or similarly to perform zero-bin subtractions [34]). With such subtractions one has

$$
\left[J_{c p^{\prime}}-J_{s}\right]=\frac{i \pi}{Q^{2}} \ln \frac{p_{-}^{\prime} \tau_{+}}{m^{2}}, \quad\left[J_{c p}-J_{s}\right]=\frac{i \pi}{Q^{2}} \ln \frac{p_{+} \tau_{-}}{m^{2}},
$$

Notice that logarithms in (70) originates from the UV-region because IR singularities cancel in the difference exactly as it was discussed in [34. Now the sum of the all contributions reproduces the correct answer:

$$
J=\left[J_{c p^{\prime}}-J_{s}\right]+\left[J_{c p}-J_{s}\right]+J_{s}=\frac{i \pi}{Q^{2}} \ln \frac{p_{-}^{\prime} \tau_{+}}{m^{2}}+\frac{i \pi}{Q^{2}} \ln \frac{p_{+} \tau_{-}}{m^{2}}-\frac{i \pi}{Q^{2}} \ln \frac{\tau_{+} \tau_{-}}{m^{2}}=\frac{i \pi}{Q^{2}} \ln \frac{Q^{2}}{m^{2}} .
$$

One can easily see that zero-bin subtractions (69) can be also taken into account by changing the sign of the soft contribution in Eq. (62) that was noted already in [34, 46].

From considered example we can conclude, that evaluations of integrals using the method of regions in $D=4$ with specific regularization, which allows to avoid scaleless integrals, must be carried out with the proper IR-subtractions. In the dimensional regularization such subtractions as a rule are performed automatically when one neglects the scaleless integrals, see for detailed discussion [45]. 


\section{Collinear and soft contributions in two-loop integral 45}

Now we return to the calculation of the overlapping soft and collinear contributions for the more complicate two-loop integral (45). From discussion in Sec III A it is clear that the situation with the soft-collinear overlap in (45) is quite similar to the considered above one-loop example: in the 2-loop case we also expect to obtain simple a logarithm $\ln Q^{2} / m^{2}$ originating from overlap of the collinear and soft regions. This allows us to write

$$
D_{s}^{\mu_{\perp}}+\mathrm{UV} \text {-finite part }\left[D_{c p^{\prime}}^{\mu_{\perp}}+D_{c p}^{\mu_{\perp}}\right]=B_{s c}^{\mu_{\perp}} \ln \frac{Q^{2}}{m^{2}}
$$

where $B_{s c}^{\mu_{\perp}}$ denote appropriate part of the total coefficient $B^{\mu_{\perp}}$ in (46). Computing $B_{s c}^{\mu_{\perp}}$ we can follow the same line as in the one-loop case and perform all calculations in $D=4$.

In order to regularize the divergencies we introduce an infinitesimal gluon mass $\mu$. Such regularization looks quite natural in this case because the gluon mass plays the role of the virtuality cut-off for hard gluons in the hard subdiagram. Then the regularized soft contribution (48) can be written as

$$
\begin{aligned}
D_{s}^{\mu_{\perp}}=\mathcal{C} \bar{\chi}_{\boldsymbol{\beta}}\left[\gamma_{\perp}^{\mu}\right]_{\beta_{3} \alpha_{3}} & \chi_{\boldsymbol{\alpha}} \int d k_{1,2}^{+} \frac{\left[\gamma^{i}\right]_{\beta_{1} \alpha_{1}^{\prime}}\left[\gamma^{i}\right]_{\beta_{2} \alpha_{2}^{\prime}}}{\bar{y}_{3}\left[-Q\left(k_{1}^{+}+k_{2}^{+}\right)\right]\left[-y_{3} Q\left(k_{1}^{+}+k_{2}^{+}\right)-\mu^{2}\right]\left[-y_{1} Q k_{1}^{+}-\mu^{2}\right]} \\
& \times \int d k_{1,2}^{-} \frac{\left[\gamma^{j}\right]_{\beta_{1}^{\prime} \alpha_{1}}\left[\gamma^{j}\right]_{\beta_{2}^{\prime} \alpha_{2}}}{\bar{x}_{3}\left[-Q\left(k_{1}^{-}+k_{2}^{-}\right)\right]\left[-x_{3} Q\left(k_{1}^{-}+k_{2}^{-}\right)-\mu^{2}\right]\left[-x_{1} Q k_{1}^{-}-\mu^{2}\right]} \\
& \times \int d k_{12 \perp} \frac{m^{2}(1)_{\alpha_{1}^{\prime} \beta_{1}^{\prime}}(1)_{\alpha_{2}^{\prime} \beta_{2}^{\prime}}}{\left[k_{1}^{2}-m^{2}\right]\left[k_{2}^{2}-m^{2}\right]}
\end{aligned}
$$

where we used that in Breit system $p_{-}^{\prime} \simeq p_{+} \simeq Q$, see Appendix A. Calculation of these integrals is a bit tedious but follows a basic line: two integrations performed by residue and the remnant integrals can be further computed keeping the most singular at $\mu \rightarrow 0$ terms. The details can be found in Appendix D. The result reads

$$
D_{s}^{\mu_{\perp}}=\mathcal{C} \bar{\chi}_{\boldsymbol{\beta}}\left[\gamma^{i} \gamma^{j}\right]_{\beta_{1} \alpha_{1}}\left[\gamma^{i} \gamma^{j}\right]_{\beta_{2} \alpha_{2}}\left[\gamma_{\perp}^{\mu}\right]_{\beta_{3} \alpha_{3}} \chi_{\boldsymbol{\alpha}} \frac{(2 \pi i)^{2}}{Q^{6}} \frac{1}{x_{1} \bar{x}_{3}^{2}} \frac{1}{y_{1} \bar{y}_{3}^{2}}\left(1-\frac{\pi^{2}}{6}\right) \ln \frac{\tau_{+} \tau_{-}}{m^{2}}+\mathcal{O}(1)
$$

where we again used $\tau_{+}=\mu^{2} / p_{-}^{\prime}$ and $\tau_{-}=\mu^{2} / p_{+}$.

Consider now the collinear term (50). We pick up from the collinear kernel $\mathcal{V}$ only UV-finite part (UV-f.p.) relevant for our calculation. Then the collinear integral reads

$$
\text { UV-f.p. } D_{c p^{\prime}}^{\mu_{\perp}} \simeq \mathcal{C} \int d k_{1}^{-} d k_{2}^{-} \frac{\Psi_{20}^{\boldsymbol{\beta}}\left(k_{i}^{-}\right)\left[\gamma^{j}\right]_{\beta_{1} \alpha_{1}}\left[\gamma^{j}\right]_{\beta_{2} \alpha_{2}}\left[\gamma_{\perp}^{\mu}\right]_{\beta_{3} \alpha_{3}} \chi_{\boldsymbol{\alpha}}}{\left[-\bar{x}_{3} Q\left(k_{1}^{-}+k_{2}^{-}\right)\right]\left[-\bar{x}_{3} Q\left(k_{1}^{-}+k_{2}^{-}\right)-\mu^{2}\right]\left[-x_{1} Q k_{1}^{-}-\mu^{2}\right]}
$$

with

$$
\begin{aligned}
\Psi_{20}^{\boldsymbol{\beta}}\left(k_{i}^{-}\right)= & \bar{\chi}_{\beta_{1}^{\prime} \beta_{2}^{\prime} \beta_{3}}^{\prime}\left[\gamma^{i}\right]_{\beta_{1}^{\prime} \beta_{1}}\left[\gamma^{i}\right]_{\beta_{2}^{\prime} \beta_{2}} \int d k_{1}^{+} d k_{2}^{+} d k_{1 \perp} d k_{2 \perp} \frac{m^{2}}{\left[k_{2}^{2}-m^{2}\right]\left[k_{1}^{2}-m^{2}\right]} \\
& \times \frac{-2\left(p^{\prime} k_{1}\right)}{\left[\left(p^{\prime}-k_{1}-k_{2}\right)^{2}-m^{2}\right]\left[\left(p^{\prime}-p_{3}^{\prime}-k_{1}\right)^{2}-m^{2}\right]\left(k_{1}+k_{2}-p^{\prime}+p_{3}^{\prime}\right)^{2}\left(k_{1}-p_{1}^{\prime}\right)^{2}}
\end{aligned}
$$

The notation $\Psi_{20}$ is introduced taking into account the structure of DA described in Eq.(55). Recall that according to (50) the integrals in Eq. (75) represent the convolution of the hard coefficient function with the perturbative DA $\Psi_{20}$. We carried out the calculations of the integrals in Eq. (76) keeping only the most singular contributions at the limit $k_{i}^{-} \sim 0$. The details are described in Appendix E. The result can be written as

$$
\begin{aligned}
\Psi_{20}^{\boldsymbol{\beta}}\left(k_{i}^{-}\right)= & \bar{\chi}_{\beta_{1}^{\prime} \beta_{2}^{\prime} \beta_{3}}^{\prime}\left[\gamma^{i}\right]_{\beta_{1}^{\prime} \beta_{1}}\left[\gamma^{i}\right]_{\beta_{2}^{\prime} \beta_{2}} \frac{(-1)(2 \pi i)^{2}}{y_{1} \bar{y}_{3}^{2} Q^{3}} \\
& \times \theta\left(0<k_{1}^{-} / Q<k_{1 \max }^{-}\right) \theta\left(0<k_{2}^{-} / Q<k_{2 \max }^{-}\right) k_{2}^{-} \ln \left(1+\frac{k_{1}^{-}}{k_{2}^{-}}\right)+\ldots
\end{aligned}
$$


where dots denote the regular at the limit $k_{i}^{-} \sim 0$ contributions which we do not consider for simplicity; the quantities $k_{1,2 \max }^{-} \sim 1$ denote the upper boundary for the relative light-cone components $k_{i}^{-} / p_{-}^{\prime}$. Their explicit value is irrelevant because we compute the integral in Eq.(75) with the leading logarithmic accuracy. Notice that from the expression (77) one can see that the end-point behavior of $\Psi_{20}$ at small fractions vanishes linearly $\left(k_{i}^{-} \rightarrow 0\right.$ and ratio $k_{1}^{-} / k_{2}^{-}$is fixed )

$$
\Psi_{20}\left(k_{i}^{-} \rightarrow 0\right) \sim k_{2}^{-} \text {. }
$$

This behavior differs from the quadratical asymptote $\sim k_{1}^{-} k_{2}^{-}$expected from the QCD evolution. As a result the collinear convolution integral is singular as discussed in Sec.IIIA.

In order to see this we substitute (77) into (75) and compute the regularized convolution integrals. For simplicity, we shall take into account the appropriate zero-bin subtractions changing the sign of soft contribution (74) in the sum (72). Then we have

$$
\text { UV-f.p. } D_{c p^{\prime}}^{\mu_{\perp}}=\mathcal{C} \bar{\chi}_{\boldsymbol{\beta}}\left[\gamma^{i} \gamma^{j}\right]_{\beta_{1} \alpha_{1}}\left[\gamma^{i} \gamma^{j}\right]_{\beta_{2} \alpha_{2}}\left[\gamma_{\perp}^{\mu}\right]_{\beta_{3} \alpha_{3}} \chi_{\boldsymbol{\alpha}} J_{c p^{\prime}}
$$

where

$$
\begin{aligned}
J_{c p^{\prime}} & =\frac{(2 \pi i)^{2}}{Q^{6}} \frac{1}{x_{1} \bar{x}_{3}^{2}} \frac{1}{y_{1} \bar{y}_{3}^{2}} \int_{0}^{k_{1 \max }^{-}} d k_{1}^{-} \int_{0}^{k_{2 \max }^{-}} d k_{2}^{-} \frac{k_{2}^{-} \ln \left(1+k_{1}^{-} / k_{2}^{-}\right)}{\left(k_{1}^{-}+k_{2}^{-}\right)^{2}\left[k_{1}^{-}+\tau_{-} / x_{1} p_{-}^{\prime}\right]} \\
& \simeq \frac{(2 \pi i)^{2}}{Q^{6}} \frac{1}{x_{1} \bar{x}_{3}^{2}} \frac{1}{y_{1} \bar{y}_{3}^{2}} \int_{0}^{k_{1 \max }^{-}} \frac{d k_{1}^{-}}{k_{1}^{-}+\tau_{-} / x_{1} p_{-}^{\prime}} \int_{0}^{\infty} d k_{2}^{-} \frac{k_{2}^{-} \ln \left(1+1 / k_{2}^{-}\right)}{\left(1+k_{2}^{-}\right)^{2}} \\
& \simeq \frac{(2 \pi i)^{2}}{Q^{6}} \frac{1}{x_{1} \bar{x}_{3}^{2}} \frac{1}{y_{1} \bar{y}_{3}^{2}}\left(1-\frac{\pi^{2}}{6}\right) \ln \tau_{-} / p_{-}^{\prime} .
\end{aligned}
$$

The similar calculation for the second collinear integral yields

$$
\text { UV-f.p. } D_{c p}^{\mu_{\perp}}=\mathcal{C} \bar{\chi}_{\boldsymbol{\beta}}\left[\gamma^{i} \gamma^{j}\right]_{\beta_{1} \alpha_{1}}\left[\gamma^{i} \gamma^{j}\right]_{\beta_{2} \alpha_{2}}\left[\gamma_{\perp}^{\mu}\right]_{\beta_{3} \alpha_{3}} \chi_{\boldsymbol{\alpha}} \frac{(2 \pi i)^{2}}{Q^{6}} \frac{1}{x_{1} \bar{x}_{3}^{2}} \frac{1}{y_{1} \bar{y}_{3}^{2}}\left(1-\frac{\pi^{2}}{6}\right) \ln \tau_{+} / p_{+} .
$$

Substituting obtained results into (72) and changing sign in front of the soft term we obtain

$$
B_{s c}^{\mu_{\perp}} \ln \frac{Q^{2}}{m^{2}}=\mathcal{C} \bar{\chi}_{\boldsymbol{\beta}}\left[\gamma^{i} \gamma^{j}\right]_{\beta_{1} \alpha_{1}}\left[\gamma^{i} \gamma^{j}\right]_{\beta_{2} \alpha_{2}}\left[\gamma_{\perp}^{\mu}\right]_{\beta_{3} \alpha_{3}} \chi_{\boldsymbol{\alpha}} \frac{(2 \pi i)^{2}}{Q^{6}} \frac{1}{x_{1} \bar{x}_{3}^{2}} \frac{1}{y_{1} \bar{y}_{3}^{2}}\left(1-\frac{\pi^{2}}{6}\right) \ln \frac{m^{2}}{Q^{2}}
$$

We see that all $\tau$-regulators cancel as they should and we obtain a simple large logarithm. Recall that the collinear contributions are associated with the hard rescattering term $F_{1}^{(h)}$. Therefore this calculation explicitly demonstrates that the soft and hard spectator scattering contributions are related and must be computed consistently.

To summarize this section, we demonstrated that the 2-loop diagram with massive quarks has a large logarithmic term originating from the overlap of the soft and collinear regions. The appearance of this logarithm does not contradict to the discussed factorization scheme. We demonstrated that in the perturbation theory one can perform the consistent description of large- $Q$ asymptotic of two-loop diagrams using definitions of SCF (14) and DA (44) with collinear quarks instead of nucleon state. We have also seen that collinear integrals in the hard spectator contribution must be singular and such situation can be realized only due to to the specific end-point behavior of the perturbative DA.

From this lesson we can suggest a general solution of the problem with the end-point divergencies in the soft spectator scattering contribution obtained in Sec.II in the perturbation theory. The soft spectator scattering together with chiral symmetry breaking by quark mass $m$ in QCD make possible the soft collinear overlap and as a result the violation of the collinear factorization for the $\mathrm{FF} F$. In this case the perturbative DA $\boldsymbol{\Psi}\left(x_{i}, \mu\right)$ at low normalization point $\mu=m$ has a such end-point behavior which leads to the end-point singularities in the collinear convolution integrals. At the same time the convolution integral of the hard kernel with the collinear one remains well defined. Such mechanism is not suppressed by inverse power of $Q$ because the quark mass is relevant only for in definition of the soft correlation function.

This allows us to expect that the factorization of the realistic hard $F_{1}^{(h)}$ and soft $F_{1}^{(s)}$ contributions in the requires an additional regularization for the separation of collinear and soft sectors. Such separation has been carried out for the case of 2-loop diagram but a realization of similar scheme for physical form factor involving the different nonperturbative matrix elements requires farther work. 


\section{PHENOMENOLOGICAL APPLICATIONS OF SCET FACTORIZATION}

From the consideration of Secs. II and III we can conclude that the practical realization of the factorization scheme discussed in the introduction has some difficulties. If we assume that the nucleon DA at low normalization point has specific non-asymptotic end-point behavior as discussed in Sec.III then the collinear and soft convolution integrals are not defined in the both equations (2) and (3). Such situation in a some sense is similar to the semi-inclusive factorization with the transverse-momentum dependent PDFs, see e.g. [48] : at a formal level we have established all the dominant regions and provided the formal definitions for the all nonperturbative quantities but that is not enough. The complication arises due to the overlap of the collinear and soft regions and, as a result, appearance of the large rapidity $\operatorname{logarithms} \sim \ln Q / \Lambda$. This is well known problem, for many processes where collinear factorization is broken due to singular end-point behavior of the convolution integrals see, e.g., 34, 35, 49 51]. The same situation is also relevant for many other hadronic reactions see, for instance, recent discussions in [52 55] and references there in. The specific of the FF factorization is that the soft functions defined in SCET involve not only Wilson lines but also quark fields, appearing as a soft spectators. Therefore there are two possible configurations $F_{1}^{(s)}$ and hard $F_{1}^{(h)}$ associated with the different hard subprocesses.

Usually the presence of two hard scales: the hard $\sim Q^{2}$ and hard-collinear $\sim \Lambda Q$ in the description of nucleon FFs allows one to perform the factorization in two steps. First, one integrates over the hard fluctuations and passes from QCD to SCET-I. This effective theory includes hard-collinear modes which can be further factorized if the virtualities of the hard-collinear particles are quite large. Integrating over hard-collinear modes one passes from SCET-I to SCET-II which includes only collinear and soft particles with the virtualities of order $\Lambda^{2}$. However if the value of $Q$ is moderate, (for instance, the hard-collinear scale $Q \Lambda \sim m_{N} \sim 1 \mathrm{GeV}^{2}$ is not large in order to serve as expansion parameter) then one can not perform SCET-II factorization. Phenomenologically such situation is relevant for quite a large range of experimentally accessible values of momentum transfer. For instance, if $Q^{2}$ is in the range of $4-25 \mathrm{GeV}^{2}$ one obtains that $Q \Lambda$ varies between $0.8-2 \mathrm{GeV}^{2}$ assuming that $\Lambda \simeq 400 \mathrm{MeV}$. Taking into account that QCD expansion of the jet functions is starting from $\alpha_{s}^{2}$ we can expect that one needs much larger hardcollinear scale in order to see the dominance of this leading contribution. On the other hand we can not ignore that the soft contribution $F_{1}^{(s)}$ is suppressed by a Sudakov form factor. If this suppression is strong enough then then $F_{1}^{(s)}$ would be subleading compared to the hard spectator contribution $F_{1}^{(h)}$. However many phenomenological studies indicate that in the intermediate region of $Q^{2}$ such suppression is still weak and the soft mechanism provides essential contribution to the physical FFs. May be heuristically this can be explained also by the relative smallness of $F_{1}^{(h)}$ which already at leading order is suppressed as $\alpha_{s}^{2}\left(Q^{2}\right)$. Therefore in order to estimate the relative importance of the two terms it is necessary to include both of them consistently within SCET-I factorization scheme. But the end-point singularities in the hard scattering contribution make such program complicates such program. Such situation is relevant also for $F_{2}$ and for many other hard exclusive reactions involving nucleons. In some cases this difficulty can be avoided using the universality of the SCET-I matrix elements.

Suppose that we have different scattering processes which are described within SCET-I factorization and depend on the same SCET-I matrix element. Using universality of the SCET-I amplitude one can define the so-called physical subtraction scheme [56, 57] which allows one to perform the systematic calculations of the hard spectator scattering contributions associated with the symmetry breaking corrections. The idea of this approach is very simple: the SCET-I soft-overlap form factor or amplitude can be rewritten as a sum of one of the physical amplitudes and the corresponding hard spectator contribution. Then this combination can be used further for the analysis of physical amplitudes of other processes with the same SCET-I matrix elements. After such redefinition the end-point singularities in the combination of the hard spectator terms must cancel and one obtains the well defined hard correction. This scheme has been successfully used for analysis of different B-meson decay amplitudes and we expect that it can also be used for the analysis of the different hadronic reactions with the soft spectator scattering contributions.

Let us illustrate the above discussion by one concrete example. Consider the following processes: $\gamma^{*} N \rightarrow N$ describing proton and neutron form factors at large $Q^{2}$ and $\gamma^{*} p \rightarrow \pi^{0} p$ describing wide-angle hard electroproduction of pion with $s, t, Q^{2} \gg \Lambda^{2}$. We suppose that nucleon form factors are described by following tentative formulae:

$$
\begin{aligned}
& F_{1}^{p}(Q)=C_{A}(Q)\left\{e_{u} f_{1}^{\mathrm{u}}(Q)+e_{d} f_{1}^{\mathrm{d}}(Q)\right\}+\boldsymbol{\Psi} * \mathbf{H}_{p} * \boldsymbol{\Psi}, \\
& F_{1}^{n}(Q)=C_{A}(Q)\left\{e_{u} f_{1}^{\mathrm{d}}(Q)+e_{d} f_{1}^{\mathrm{u}}(Q)\right\}+\boldsymbol{\Psi} * \mathbf{H}_{n} * \boldsymbol{\Psi},
\end{aligned}
$$


where we used definitions (201),(21) and isotopic symmetry, symbols $\mathbf{H}_{p, n}$ denote the hard scattering kernel for the proton and nucleon cases, respectively. We also assume that the convolution integrals denoted by asterisk are regularized using some IR-regulator. Solving these equations with respect to SCET FFs one finds $\left(e_{u}=2 / 3, e_{d}=-1 / 3\right)$

$$
\begin{gathered}
f_{1}^{\mathrm{u}}=C_{A}^{-1}\left\{2 F_{1}^{p}+F_{1}^{n}-\mathbf{\Psi} *\left(2 \mathbf{H}_{p}+\mathbf{H}_{n}\right) * \mathbf{\Psi}\right\}, \\
f_{1}^{\mathrm{d}}=C_{A}^{-1}\left\{F_{1}^{p}+2 F_{1}^{n}-\mathbf{\Psi} *\left(\mathbf{H}_{p}+2 \mathbf{H}_{n}\right) * \mathbf{\Psi}\right\},
\end{gathered}
$$

The pion production process can also be described as a sum of two contributions as shown in Fig 6 . So
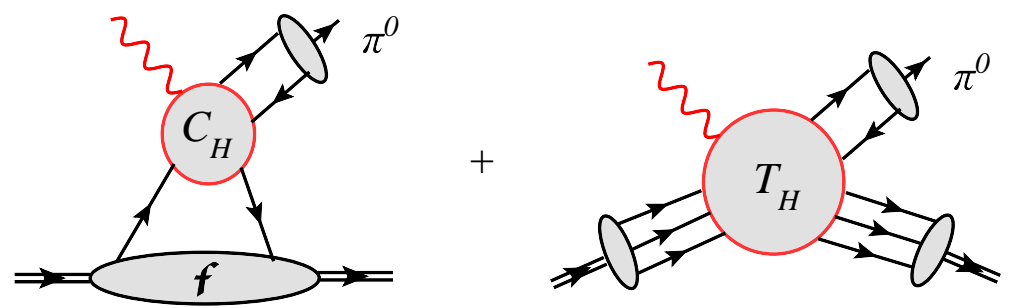

FIG. 6: The sum of the soft and hard spectator contributions for the wide-angle hard pion electroproduction

far we are not going to prove the exact factorization theorem for this process. Most important for us is that this configuration can be considered as one possible contribution to the nucleon helicity conserving amplitudes $A_{i}^{\pi^{0}}$. The first term in Fig $[$ describes the soft overlap nucleon contribution and can be expressed in terms of the same SCET-I form factor $f_{1}^{u, d}$. The pion blob is described by pion distribution amplitudes. The second contribution in Fig $[$ can be associated with the hard spectator scattering and it is described as usually as a convolution of nucleon and pion DAs with the hard scattering kernel $T_{H}$. Therefore schematic expression of the contribution in Fig $[$ reads

$$
A_{i}^{\pi^{0}}=\varphi_{\pi}(z) * C_{H}(z)\left(e_{u} f_{1}^{\mathrm{u}}-e_{d} f_{1}^{\mathrm{d}}\right)+\mathbf{\Psi}\left(x_{i}\right) * \varphi_{\pi}(z) * \mathbf{T}_{H}\left(x_{i}, y_{i}, z\right) * \mathbf{\Psi}\left(y_{i}\right)
$$

where we again assume a some regularization for the divergent convolution integrals. Substitution of expressions for the SCET form factors (87) and (88) in Eq.(89) yields

$$
\begin{aligned}
A_{i}^{\pi^{0}} & =\varphi_{\pi}(z) * C_{H}(z) / C_{A} \frac{1}{3}\left(5 F_{1}^{p}+4 F_{1}^{n}\right) \\
& +\boldsymbol{\Psi}\left(x_{i}\right) * \varphi_{\pi}(z) *\left(\mathbf{T}_{H}\left(x_{i}, y_{i}, z\right)-C_{H}(z) / C_{A} \frac{1}{3}\left\{5 \mathbf{H}_{p}\left(x_{i}, y_{i}\right)+4 \mathbf{H}_{n}\left(x_{i}, y_{i}\right)\right\}\right) * \mathbf{\Psi}\left(y_{i}\right) .
\end{aligned}
$$

Thus the soft overlap contribution is represented in terms of the physical FFs $F_{1}^{p, n}$. The ratio $C_{H}(z) / C_{A}$ depends only from the factorization scale associated with the evolution of pion DA. All Sudakov logarithms must cancel in this ratio. On the other hand, the end-point singularities in the hard scattering kernels must be compensated in the combination of $\mathbf{T}_{H}$ and $\mathbf{H}_{n, p}$ in rhs of (91). The simple analysis show that the hard spectator correction is of order $\alpha_{s}^{3}$. At the same time the ratio $C_{H}(z) / C_{A}=\alpha_{s} C_{\mathrm{LO}}(z)+\mathcal{O}\left(\alpha_{s}^{2}\right)$. The next-to-leading contribution can be computed from the one loop corrections to $C_{H}(z)$ and $C_{A}$ and the hard spectator corrections can appear only in the next-next-to-leading order.

Therefore with this example we demonstrated how the SCET-I factorization on the soft and hard spectator contributions allows one to analyze the realistic hadronic processes at the intermediate momentum transfer. Of course, such method can be used when the number of observables is larger then the number of unknown SCET amplitudes. The more detailed application of this scheme for analysis of different processes we are going to present in the separate publication.

If the values of $Q$ are quite large so that the hard-collinear scale is already a good parameter for the asymptotic approximation one can try to perform the second factorization step and pass from the intermediate SCET-I to the low energy SCET-II. Performing this matching one must provide a solution of the soft collinear overlap in order to treat correctly corresponding large logarithms and avoid a double counting between the soft and hard spectator scattering contributions. 
Formulation of such scheme is a difficult task and at present time we have not yet found a convincing technical realization of this idea which can be realized in our case. The most challenging problem is the consistent formulation of the zero-bin (or infrared) subtractions for the collinear convolutions integrals with nonpertubative DAs (or other collinear matrix elements) and corresponding UV-renormalization of the soft rescattering contribution involving soft correlation functions, i.e. unambiguous matching of collinear and soft modes and consequently the definition of the hard and soft rescattering contributions. The other difficult moment is the absence of a well-defined regularization. Dimensional regularization can not be used for the calculation of the soft convolution integrals because corresponding integrands are scaleless. Therefore one has to invent the other method, for instance off-shell momenta or analytic regularization. As a rule, such regularization has potential problems beyond the leading order that makes difficult systematic calculations and analysis. Recently several ideas has been suggested see, e.g., 53 55] in the framework of SCET approach which can potentially solve these problems. We leave the investigation of this question for future work.

\section{DISCUSSION}

In this paper we investigated the large- $Q$ asymptotic form of the proton $\mathrm{FF} F_{1}$ using factorization approach. In our previous publication [1] we suggested that the full factorization formula for the nucleon FF $F_{1}$ is given by the sum of two contributions: hard spectator scattering term $F_{1}^{(h)}$ and the second term describing the scattering with the soft spectator quarks which we refer as the soft spectator contribution $F_{1}^{(s)}$.

In the first publication [1] we provided an explicit definition for the leading power contribution $F_{1}^{(s)}$ in terms of the hard and hard-collinear coefficient functions and nonpertubative matrix elements. In present paper we carried out more detailed analysis of suggested factorization. We computed the leading order jet functions $J$ appearing in the matching from SCET-I to SCET-II. Using this result we obtained that the corresponding soft convolution integrals in definition $F_{1}^{(s)}(\underline{3})$ have logarithmic divergency at the end-point region. This observation follows from the boost invariance and therefore does not depend on any model assumptions about the soft correlation function $\boldsymbol{S}$ in Eq.(3).

We suggested that the appearance of end-point singularity can be explained by overlap of collinear and soft regions. In order to verify this supposition we carried out analysis of two-loop QCD diagrams with massive quarks and investigated the dominant contributions associated with the different regions. In this case the non-perturbative matrix elements associated with the soft correlation function and distribution amplitudes can be computed explicitly in the perturbation theory. We found that the overlap of the soft and collinear sectors provides the large rapidity logarithm $\sim \ln Q / m$ where $m$ is the quark mass which plays the role of the soft scale. Thus the logarithmic divergence of the soft convolution integrals can be naturally explained by the overlap with the collinear region. In such case the collinear convolution integrals describing the hard spectator scattering has also end-point singularity and can not be well defined without special regularization. The latter allows us to conclude that the separation of the hard and soft spectator contributions for the FF $F_{1}$ is closely related to the problem of separation of soft and collinear sectors in SCET.

At first glance such situation looks controversial for the $\mathrm{FF} F_{1}$ taking into account the end-point behavior of the nucleon DA which is usually estimated from the QCD evolution. The eigenfunctions of the the leading order evolution kernel vanish quite rapidly at the end-point region that ensures a good convergence of the collinear integrals defining the hard spectator contribution. However the soft spectator scattering and explicit chiral symmetry breaking (mass of the soft quarks) in perturbative calculations provides the mechanism which makes possible the soft collinear overlap and violation of the collinear factorization without any conflict with these arguments. QCD evolution is not sensitive to the effects related to the mass of quarks and therefore in this case it can not provide a signal about the problem with the end-point behavior of the collinear convolution integrals. Formally, in the perturbation theory this scenario is realized as following. The end-point behavior of the DA at low normalization $\left(\mu_{F}=m\right)$ is different from the one which we expect from its evolution: it vanishes more slowly. Then the convolution integral with such DA has logarithmic end-point divergency.

Extrapolating these arguments beyond the perturbation theory we can not use the mass of the soft quarks as realistic argument anymore. But on the other hand we can expect that the nontrivial contribution to the soft correlation function $\boldsymbol{S}$ can be obtained due to the dynamical chiral symmetry breaking 
in non-perturbative QCD. In that case one can have the nontrivial soft spectator scattering contribution which has the end-point singularities. Basing on this observation we can formulate the following conjecture. The soft spectator scattering and chiral symmetry breaking in non-perturbative QCD make possible the soft collinear overlap and as a result violation of the collinear factorization for FF $F_{1}$. This leads to the appearing of the end-point singularities in the soft and hard spectator scattering contributions. In this case the nucleon $D A \varphi_{N}\left(x_{i}, \mu_{0}\right)$ at a low normalization point $\mu_{0} \sim \Lambda$ vanishes more slowly then the asymptotic shape and such behavior leads to the end-point singularities in the collinear convolution integrals. Such a behavior is very important for the consistent description of the large rapidity logarithm appearing due to overlap of the hard and soft sectors. The end-point singularity arising in the soft spectator contribution can be considered as strong argument in support of such scenario.

We expect that the hypothesis about the role of chiral symmetry breaking in the non-perturbative calculation of the soft correlation function might be verified in different model calculations. A certain indication about the non-asymptotic end-point behavior of the nucleon DA was already obtained from the non-perturbative calculations. In work [41] the nucleon DA $\varphi_{N}\left(z_{i}\right)$ has been computed in the chiral quark-soliton model [42, 43] using large $N_{c}$ approximation. The DA is estimated at $\mu_{0} \leq 600 \mathrm{MeV}$ and it was found that it does not vanish at the end-point limit if the one of the fractions, for instance, $x_{1}$ is fixed and $x_{2} \rightarrow 0$. Unfortunately the region of small fractions in [41] have not been studied analytically but the authors do not exclude the linear asymptotic if the all fractions are small $x_{i} \rightarrow 0$ but their ratio is fixed [44]. On the other hand in [41] it is also noted that obtained results are valid only in the region of relatively large collinear fractions where $x_{i} N_{c} \sim 1$. It means that for the realistic value $N_{c}=3$ one can expect large $1 / N_{c}$ corrections in region with small fractions and therefore the obtained leading $1 / N_{c}$ results can be modified.

Basing on our assumption we expect that the general scheme as described in Eqs (2)-(3) is valid but the explicit calculation of the soft $F_{1}^{(s)}$ and hard $F_{1}^{(h)}$ spectator contributions requires a certain prescription for separation of soft and collinear degrees of freedom. The collinear convolution integrals in the $F_{1}^{(h)}$ must be regularized and corresponding large rapidity logarithms if possible must be also resummed. In present paper we do not provide any systematic formalism for the factorization of the soft and collinear degrees of freedom. However, we would like to stress that the soft spectator contribution plays the important role in the correct description of such factorization.

Our analysis provides additional arguments in support of the phenomenological descriptions which assumes that at some moderate values of $Q$ the dominant contribution is provided by soft-overlap contribution which can be associated with the soft spectator scattering see, e.g., 23 26]. In spite of the factorization complexities the SCET provides a powerful framework for investigation of other hard exclusive reactions. SCET description of the soft spectator contribution naturally introduces a concept of two large scales: hard $\sim Q^{2}$ and hard-collinear $\sim \Lambda Q$. This naturally defines the region of moderate values of large momentum transfer $Q$ : it corresponds to the situation when the inverse power of hard scale $1 / Q^{2}$ is a good expansion parameter but the hard-collinear scale is still not too large. Taking $\Lambda \simeq 300-400 \mathrm{MeV}$ and $Q^{2}=25 \mathrm{GeV}^{2}$ one easily obtains that $Q \Lambda \simeq 1.5-2 \mathrm{GeV}^{2}$. Hence in the situation when $Q^{2} \simeq 10-20 \mathrm{GeV}^{2}$ which overlaps with the majority of existed and upcoming experiments, one can perform consistently only SCET-I factorization. Within such factorization scheme it is useful to take into account both soft and hard spectator scattering contributions because their relative contribution might be comparable if the suppression from the Sudakov form factor for the soft-overlap term is not sufficiently large. Using the universality of the SCET FFs one can make the combined analysis of the different processes and check the relevance of the soft overlap contribution in a model independent way. Technically it can be done using the, so-called, physical subtraction scheme which allows to to perform a systematic consideration and also solves the problem with the end-point singularities from the hard spectator corrections. In particular, such analysis might be very interesting for hard exclusive processes with baryons, such as wide-angle Compton scattering, wide-angle meson production and their timelike analogs. This work is in progress.

\section{Acknowlegmens}

This work was supported by the Helmholtz Institute Mainz. The author is grateful to V. Pascalutsa and M. Venderhaeghen for reading the manuscript. 


\section{Appendix A: Brief summary of used notations}

Through the paper we imply Breit frame

$$
q=p^{\prime}-p=Q\left(\frac{n}{2}-\frac{\bar{n}}{2}\right), \quad n=(1,0,0,-1), \bar{n}=(1,0,0,1), \quad(n \cdot \bar{n})=2,
$$

and define the external momenta as

$$
\begin{gathered}
p=\mathcal{Q} \frac{\bar{n}}{2}+\frac{m_{N}^{2}}{\mathcal{Q}} \frac{n}{2}, \quad p^{\prime}=\mathcal{Q} \frac{n}{2}+\frac{m_{N}^{2}}{\mathcal{Q}} \frac{\bar{n}}{2}, \quad \mathcal{Q}=Q \frac{1}{2}\left[1+\sqrt{1+\frac{4 m_{N}^{2}}{Q^{2}}}\right]=Q+\mathcal{O}\left(m_{N}^{2} / Q^{2}\right), \\
2\left(p p^{\prime}\right)=\mathcal{Q}^{2}+\frac{m_{N}^{4}}{\mathcal{Q}^{2}} \approx Q^{2},
\end{gathered}
$$

where $m_{N}$ is the nucleon mass. For the incoming and outgoing collinear quarks we always imply

$$
p_{i}=x_{i} \mathcal{Q} \frac{\bar{n}}{2}+p_{\perp i}+\left(x_{i}^{\prime} \frac{m_{N}^{2}}{\mathcal{Q}}\right) \frac{n}{2}, \quad p_{i}^{\prime}=y_{i} \mathcal{Q} \frac{n}{2}+p_{\perp i}^{\prime}+\left(y_{i}^{\prime} \frac{m_{N}^{2}}{\mathcal{Q}}\right) \frac{\bar{n}}{2},
$$

with the transverse momenta

$$
p_{\perp}^{2} \sim p_{\perp}^{\prime 2} \sim \Lambda^{2}
$$

and where $x_{i}$ and $x_{i}^{\prime}$ denote fractions of the corresponding momentum-component. Computing the Feynman diagrams in Sec.III we neglect by power suppressed components and assume

$$
p \simeq Q \frac{\bar{n}}{2}, p_{i} \simeq x_{i} p, \quad p^{\prime} \simeq Q \frac{n}{2}, \quad p_{i}^{\prime} \simeq y_{i} p^{\prime},
$$

In many formulas we use convenient notation $\bar{x}_{i}=1-x_{i}$. We also use the following notation for scalar products

$$
(a \cdot n) \equiv a_{+},(a \cdot \bar{n}) \equiv a_{-} .
$$

and Dirac contractions

$$
p_{\mu} \gamma^{\mu} \equiv \not p \equiv \hat{p} .
$$

Nucleon FFs are defined as the matrix elements of the e.m. current between the nucleon states:

$$
\left\langle p^{\prime}\left|J_{e . m .}^{\mu}(0)\right| p\right\rangle=\bar{N}\left(p^{\prime}\right)\left[\gamma^{\mu}\left(F_{1}+F_{2}\right)-\frac{\left(p+p^{\prime}\right)^{\mu}}{2 m_{N}} F_{2}\right] N(p),
$$

with nucleon spinors normalized as $\bar{N} N=2 m_{N}$.

\section{Appendix B: Soft correlation function in perturbation theory}

The leading order perturbative expression for the $\boldsymbol{S}_{V}^{\mathrm{ud}}$ reads:

$$
\begin{aligned}
\left(\boldsymbol{S}_{V}^{\mathrm{ud}}\right)_{\mathrm{LO}}=\frac{3}{16 \pi^{8}} \int d k_{1} d k_{2} \delta\left(k_{1}^{+}-\omega_{1}\right) & \delta\left(k_{2}^{+}-\omega_{2}\right) \delta\left(k_{1}^{-}-\nu_{1}\right) \delta\left(k_{2}^{-}-\nu_{2}\right) \\
& \frac{\frac{1}{8} \operatorname{Tr}\left[\left(\hat{k}_{1}+m\right) \gamma_{-} C\left(C \gamma_{+}\left(\hat{k}_{2}+m\right)\right)^{\mathrm{T}}\right]}{\left[\omega_{1} \nu_{1}-k_{1 \perp}^{2}-m^{2}+i \varepsilon\right]\left[\omega_{2} \nu_{2}-k_{2 \perp}^{2}-m^{2}+i \varepsilon\right]}
\end{aligned}
$$


The factor $1 / 8$ in front of trace is chosen for convenience. Calculation of the trace in the numerator yields:

$$
\frac{1}{8} \operatorname{Tr}\left[\left(\hat{k}_{1}+m\right) \gamma_{-} C\left(C \gamma_{+}\left(\hat{k}_{2}+m\right)\right)^{\mathrm{T}}\right]=-m^{2}+\left(k_{1 \perp} \cdot k_{2 \perp}\right)
$$

This allows us to write:

$$
\left(\boldsymbol{S}_{V}^{\mathrm{ud}}\right)_{\mathrm{LO}}=-\frac{3 m^{2}}{16 \pi^{8}} \frac{1}{4} \int d k_{1 \perp} d k_{2 \perp} \frac{1}{\left[\omega_{1} \nu_{1}-k_{1 \perp}^{2}-m^{2}+i \varepsilon\right]\left[\omega_{2} \nu_{2}-k_{2 \perp}^{2}-m^{2}+i \varepsilon\right]} .
$$

In order to proceed further we must take into account the specific properties of the jet functions. We always assume that the soft fractions $\omega_{i}$ and $\nu_{i}$ are positive. Mathematically it comes out from the analytical properties of the diagrams and imposes specific restrictions on the integrand in Eq.(104). In order to see this assume that $-\infty<\omega_{i}<\infty$ but then we keep the Feynman $i \varepsilon$-prescription in the jet functions. From calculations of the diagrams in Fig 2 one can easily obtain that all denominators of the jet functions in Eqs. (30)-(33) are defined with $-i \varepsilon$, for instance

$$
\frac{1}{\omega_{1}+\omega_{2}} \frac{1}{\omega_{1}} \frac{1}{\omega_{2}} \rightarrow \frac{1}{\left[\omega_{1}+\omega_{2}-i \varepsilon\right]} \frac{1}{\left[\omega_{1}-i \varepsilon\right]} \frac{1}{\left[\omega_{2}-i \varepsilon\right]} .
$$

The same arguments also true for $\nu_{i}$. Consider now the convolution integrals

$$
\int d \omega_{1} d \omega_{2} \frac{1}{\left[\omega_{1}+\omega_{2}-i \varepsilon\right]} \frac{1}{\left[\omega_{1}-i \varepsilon\right]} \frac{1}{\left[\omega_{2}-i \varepsilon\right]} S_{V}\left(\omega_{i}\right),
$$

where $S_{V}\left(\omega_{i}\right)$ is represented by expression (104). Computing $d \omega_{i}$ by residues we obtain that nontrivial results originates only from the poles of the propagators in $S_{V}\left(\omega_{i}\right)$ in (104). Therefore this allows to us to represent the propagators in (104) as $\delta$-functions:

$$
\begin{aligned}
\frac{1}{\left[\omega_{1} \nu_{1}-k_{1 \perp}^{2}-m^{2}+i \varepsilon\right]\left[\omega_{2} \nu_{2}-k_{2 \perp}^{2}-m^{2}+i \varepsilon\right]}= & (2 \pi i)^{2} \theta\left(\omega_{i}>0\right) \theta\left(\nu_{i}>0\right) \\
& \times \delta\left(\omega_{1} \nu_{1}-k_{1 \perp}^{2}-m^{2}\right) \delta\left(\omega_{2} \nu_{2}-k_{2 \perp}^{2}-m^{2}\right) .
\end{aligned}
$$

Then we obtain

$$
\begin{aligned}
\left(\boldsymbol{S}_{V}^{\mathrm{ud}}\right)_{\mathrm{LO}} & =\frac{3 m^{2}}{16 \pi^{6}} \theta\left(\nu_{i}>0\right) \int d k_{1 \perp} d k_{2 \perp} \delta\left(\omega_{1} \nu_{1}-k_{1 \perp}^{2}-m^{2}\right) \delta\left(\omega_{2} \nu_{2}-k_{2 \perp}^{2}-m^{2}\right) \\
& =\frac{3 m^{2}}{16 \pi^{6}} \theta\left(\nu_{i}>0\right) \theta\left(\omega_{i}>0\right) \theta\left(\omega_{1} \eta_{1}>m^{2}\right) \theta\left(\omega_{2} \eta_{2}>m^{2}\right) .
\end{aligned}
$$

Appendix C: Derivation of the collinear contribution $D_{c p^{\prime}}^{\mu \perp}$

Consider first denominator (45). In the collinear region (49) we obtain:

$$
\begin{aligned}
& {\left[\left(p-k_{1}-k_{2}\right)^{2}-m^{2}\right]\left(p-p_{3}-k_{1}-k_{2}\right)^{2}\left(p-p_{3}-k_{1}\right)^{2}\left[\left(p_{1}-k_{1}\right)^{2}-m^{2}\right]} \\
& \simeq\left[-Q\left(k_{1}^{-}+k_{2}^{-}\right)\right]\left[-Q \bar{x}_{3}\left(k_{1}^{-}+k_{2}^{-}\right)\right]\left[-Q \bar{x}_{3} k_{1}^{-}\right]\left[-Q x_{1} k_{1}^{-}\right] \\
& \simeq Q^{4}\left(\bar{x}_{3}^{2} x_{1}\right)\left[-\left(k_{1}^{-}+k_{2}^{-}\right)\right]^{2}\left[-k_{1}^{-}\right]^{2} .
\end{aligned}
$$

The remaining propagators are soft, of order $\Lambda^{2}$. Hence for the denominator we obtain

$$
\text { Den } \sim Q^{8} \Lambda^{12}
$$

In the numerator we have

$$
\begin{aligned}
& \mathrm{Num}=\bar{\xi}_{1}^{\prime}\left(\hat{k}_{1}+m\right)\left[\begin{array}{ll}
\gamma^{j} & \xi_{1}
\end{array}\right] \bar{\xi}_{2}^{\prime} \gamma^{i}\left(\hat{p}^{\prime}-\hat{p}_{3}^{\prime}-\hat{k}_{1}+m\right) \gamma^{\alpha}\left(\hat{k}_{2}+m\right)\left[\gamma^{\beta}\left(\hat{p}-\hat{p}_{3}-\hat{k}_{1}\right) \gamma^{j} \xi_{2}\right] \\
& \bar{\xi}_{3}^{\prime} \gamma^{\alpha}\left(\hat{p}^{\prime}-\hat{k}_{1}-\hat{k}_{2}+m\right)\left[\gamma_{\perp}^{\mu}\left(\hat{p}-\hat{k}_{1}-\hat{k}_{2}+m\right) \gamma^{\beta} \xi_{3}\right]
\end{aligned}
$$


where we single out by brackets $[. .$.$] the numerators of the hard propagators and hard gluon vertices.$ Using that $\hat{p} \xi \simeq 0$ we can rewrite this piece as:

$$
\begin{aligned}
{\left[\gamma^{j} \xi_{1}\right] \otimes\left[\gamma^{\beta}\left(\hat{p}-\hat{p}_{3}-\hat{k}_{1}\right) \gamma^{j} \xi_{2}\right] } & \otimes\left[\gamma_{\perp}^{\mu}\left(\hat{p}-\hat{k}_{1}-\hat{k}_{2}+m\right) \gamma^{\beta} \xi_{3}\right] \\
& \simeq(-4)\left(p k_{1}\right)\left[\gamma^{j} \xi_{1}\right] \otimes\left[\gamma^{j} \xi_{2}\right] \otimes\left[\gamma_{\perp}^{\mu} \xi_{3}\right]
\end{aligned}
$$

Then we obtain

$$
\begin{gathered}
\operatorname{Num}=2 Q\left(-k_{1}^{-}\right) \bar{\xi}_{1}^{\prime} \gamma^{i}\left(\hat{k}_{1}+m\right)\left[\gamma^{j} \xi_{1}\right] \bar{\xi}_{2}^{\prime} \gamma^{i}\left(\hat{p}^{\prime}-\hat{p}_{3}^{\prime}-\hat{k}_{1}+m\right) \gamma^{\alpha}\left(\hat{k}_{2}+m\right)\left[\begin{array}{ll}
\gamma^{j} & \xi_{2}
\end{array}\right] \\
\times \bar{\xi}_{3}^{\prime} \gamma^{\alpha}\left(\hat{p}^{\prime}-\hat{k}_{1}-\hat{k}_{2}+m\right)\left[\gamma_{\perp}^{\mu} \xi_{3}\right] .
\end{gathered}
$$

Recall that $k_{1}^{-} \sim Q$, then for the remaining terms one finds

$$
\begin{aligned}
\bar{\xi}_{1}^{\prime} \gamma^{i}\left(\hat{k}_{1 \perp}\right)\left[\gamma^{j} \xi_{1}\right] \bar{\xi}_{3}^{\prime} \gamma^{\alpha} & \left(-\hat{k}_{1 \perp}-\hat{k}_{2 \perp}\right)\left[\gamma_{\perp}^{\mu} \xi_{3}\right] \bar{\xi}_{2}^{\prime} \gamma^{i}\left(-\hat{k}_{1 \perp}\right) \gamma^{\alpha}\left(\hat{k}_{2 \perp}\right)\left[\gamma^{j} \xi_{2}\right] \\
& \sim \Lambda^{4} \bar{\xi}_{1}^{\prime} \Gamma_{1} \xi_{1} \bar{\xi}_{2}^{\prime} \Gamma_{2} \xi_{2} \bar{\xi}_{3}^{\prime} \Gamma_{3} \xi_{3},
\end{aligned}
$$

where $\Gamma_{i}$ denote certain Dirac matrices. Hence one obtains:

$$
\text { Num } \sim Q^{2} \Lambda^{4},
$$

and for the whole diagram one finds:

$$
D_{c p^{\prime}} \sim \frac{1}{Q^{6}} \bar{\xi}_{1}^{\prime} \Gamma_{1} \xi_{1} \bar{\xi}_{2}^{\prime} \Gamma_{2} \xi_{2} \bar{\xi}_{3}^{\prime} \Gamma_{3} \xi_{3} .
$$

Taking into account notation in Eqs.(43/44) the collinear contribution can be represented as

$$
D_{c p^{\prime}}^{\mu_{\perp}} \simeq \mathcal{C} \bar{\chi}_{\boldsymbol{\beta}}^{\prime} \int d k_{1}^{-} d k_{2}^{-} \mathcal{V}_{\boldsymbol{\beta} \boldsymbol{\beta}^{\prime}}\left(k_{i}^{-}\right) \frac{\left[\gamma^{j}\right]_{\beta_{1}^{\prime} \alpha_{1}}\left[\gamma^{j}\right]_{\beta_{2}^{\prime} \alpha_{2}}\left[\gamma_{\perp}^{\mu}\right]_{\beta_{3}^{\prime} \alpha_{3}}}{\bar{x}_{3}^{2} x_{1}\left[-Q\left(k_{1}^{-}+k_{2}^{-}\right)\right]^{2}\left[-Q k_{1}^{-}\right]} \chi_{\boldsymbol{\alpha}}
$$

with

$$
\begin{aligned}
\mathcal{V}\left(k_{i}^{-}\right)= & \frac{1}{2} \int d k_{1}^{+} d k_{2}^{+} d k_{1 \perp} d k_{2 \perp} \frac{\left\{\gamma^{i}\left(\hat{k}_{1}+m\right)\right\}_{\beta_{1} \beta_{1}^{\prime}}}{\left[k_{2}^{2}-m^{2}\right]\left[k_{1}^{2}-m^{2}\right]} \\
& \times \frac{\left\{\gamma^{i}\left(\hat{p}^{\prime}-\hat{p}_{3}^{\prime}-\hat{k}_{1}+m\right) \gamma^{\alpha}\left(\hat{k}_{2}+m\right)\right\}_{\beta_{2} \beta_{2}^{\prime}}\left\{\gamma^{\alpha}\left(\hat{p}^{\prime}-\hat{k}_{1}-\hat{k}_{2}+m\right)\right\}_{\beta_{3} \beta_{3}^{\prime}}}{\left[\left(p^{\prime}-k_{1}-k_{2}\right)^{2}-m^{2}\right]\left[\left(p^{\prime}-p_{3}^{\prime}-k_{1}\right)^{2}-m^{2}\right]\left(k_{1}+k_{2}-p^{\prime}+p_{3}^{\prime}\right)^{2}\left(k_{1}-p_{1}^{\prime}\right)^{2}} .
\end{aligned}
$$

Eq. (123) yields the contribution to the evolution kernel at 2-loop approximation. The integral with respect to $k_{1,2}^{-}$in Eq.(122) can be interpreted as a convolution integral of leading order hard coefficient function with the given part of evolution kernel.

\section{Appendix D: Calculation of $D_{s}^{\mu_{\perp}}$}

The expression for the soft integral $J_{s}$ in Eq.(74) reads

$$
J_{s}=\frac{1}{Q^{6}} \frac{1}{x_{1} \bar{x}_{3}^{2}} \frac{1}{y_{1} \bar{y}_{3}^{2}} I_{s},
$$

with

$$
\begin{aligned}
I_{s} & =\int d k_{2 \perp} d k_{1 \perp} \int d k_{2}^{+} d k_{1}^{+} \frac{1}{\left[-k_{1}^{+}-\tau_{+} / y_{1}\right]\left[-\left(k_{1}^{+}+k_{2}^{+}\right)-\tau_{+} / \bar{y}_{3}\right]\left[-\left(k_{1}^{+}+k_{2}^{+}\right)\right]} \\
& \int d k_{2}^{-} d k_{1}^{-} \frac{1}{\left[-k_{1}^{-}-\tau_{-} / x_{1}\right]\left[-\left(k_{1}^{-}+k_{2}^{-}\right)-\tau_{-} / \bar{x}_{3}\right]\left[-\left(k_{1}^{-}+k_{2}^{-}\right)\right]} \frac{m^{2}}{\left[k_{2}^{2}-m^{2}\right]\left[k_{1}^{2}-m^{2}\right]}
\end{aligned}
$$


For simplicity let us redefine the notations as

$$
k_{i}^{+} \rightarrow \beta_{i}, k_{i}^{-} \rightarrow \alpha_{i}, d k_{i \perp} \rightarrow d k_{i}, \quad \tau_{1} \equiv \tau_{-} / x_{1}, \quad \tau_{3} \equiv \tau_{-} / \bar{x}_{3}, \tau_{1}^{\prime} \equiv \tau_{+} / y_{1}, \tau_{3}^{\prime} \equiv \tau_{+} / \bar{y}_{3} .
$$

So that

$$
\begin{aligned}
I_{s} & =\int d k_{2} d k_{1} \int d \beta_{1} d \beta_{2} \frac{1}{\left[-\beta_{1}-\tau_{1}\right]\left[-\left(\beta_{1}+\beta_{2}\right)-\tau_{3}\right]\left[-\left(\beta_{1}+\beta_{2}\right)\right]} \\
& \times \int d \alpha_{1} d \alpha_{2} \frac{1}{\left[-\alpha_{1}-\tau_{1}^{\prime}\right]\left[-\left(\alpha_{1}+\alpha_{2}\right)-\tau_{3}^{\prime}\right]\left[-\left(\alpha_{1}+\alpha_{2}\right)\right]} \frac{m^{2}}{\left[\alpha_{2} \beta_{2}-k_{2}^{2}-m^{2}\right]\left[\alpha_{1} \beta_{1}-k_{1}^{2}-m^{2}\right]} .
\end{aligned}
$$

Expressions in square brackets implies $[\ldots] \equiv[\ldots+i \varepsilon]$. Next we use the same trick as in Appendix A: we integrate over $d \alpha_{1,2}$ by residues, rewrite the poles as $\delta$-functions:

$$
\frac{1}{\left[\alpha_{2} \beta_{2}-k_{2}^{2}-m^{2}\right]\left[\alpha_{1} \beta_{1}-k_{1}^{2}-m^{2}\right]} \rightarrow(2 \pi i)^{2} \theta\left(\beta_{i}\right) \theta\left(\alpha_{i}\right) \delta\left(\alpha_{1} \beta_{1}-k_{1}^{2}-m^{2}\right) \delta\left(\alpha_{2} \beta_{2}-k_{2}^{2}-m^{2}\right)
$$

and integrate over transverse momenta. This yields

$$
\begin{aligned}
I_{s} & =(2 \pi i)^{2} m^{2} \int_{0}^{\infty} d \beta_{1} d \beta_{2} \frac{1}{\left[\beta_{1}+\tau_{1}\right]\left[\left(\beta_{1}+\beta_{2}\right)+\tau_{3}\right]\left(\beta_{1}+\beta_{2}\right)} \\
& \times \int_{0}^{\infty} d \alpha_{1} d \alpha_{2} \frac{\theta\left(\alpha_{2} \beta_{2}>m^{2}\right) \theta\left(\alpha_{1} \beta_{1}>m^{2}\right)}{\left[\alpha_{1}+\tau_{1}^{\prime}\right]\left[\left(\alpha_{1}+\alpha_{2}\right)+\tau_{3}^{\prime}\right]\left(\alpha_{1}+\alpha_{2}\right)}
\end{aligned}
$$

After simple substitutions this integral can be written as

$$
\begin{aligned}
I_{s}=(2 \pi i)^{2} m^{2} & \int_{0}^{\infty} d \beta_{2} \frac{1}{\left(1+\beta_{2}\right)} \int_{0}^{\infty} d \alpha_{1} d \alpha_{2} \frac{\theta\left(\alpha_{2} \beta_{2}>m^{2}\right) \theta\left(\alpha_{1}>m^{2}\right)}{\left(\alpha_{1}+\alpha_{2}\right)} \\
& \times \int_{0}^{\infty} d \beta_{1} \frac{1}{\left(\beta_{1}+\tau_{1}\right)\left[\beta_{1}\left(1+\beta_{2}\right)+\tau_{3}\right]} \frac{1}{\left(\alpha_{1}+\beta_{1} \tau_{1}^{\prime}\right)} \frac{1}{\left[\alpha_{1}+\alpha_{2}+\beta_{1} \tau_{3}^{\prime}\right]}
\end{aligned}
$$

Using that

$$
\begin{gathered}
\frac{\beta_{1}}{\left[\beta_{1}\left(1+\beta_{2}\right)+\tau_{3}\right]}=\frac{1}{\left(1+\beta_{2}\right)}-\frac{\tau_{3}}{\left(1+\beta_{2}\right)\left[\beta_{1}\left(1+\beta_{2}\right)+\tau_{3}\right]} \simeq \frac{1}{\left(1+\beta_{2}\right)}, \\
\frac{1}{\left(\alpha_{1}+\beta_{1} \tau_{1}^{\prime}\right)} \frac{1}{\left[\alpha_{1}+\alpha_{2}+\beta_{1} \tau_{3}^{\prime}\right]} \\
=\frac{1}{\left(\alpha_{1}+\alpha_{2}\right)}\left[\frac{1}{\left(\alpha_{1}+\beta_{1} \tau_{1}^{\prime}\right)}-\frac{\tau_{3}^{\prime}}{\left[\alpha_{1}+\alpha_{2}+\beta_{1} \tau_{3}^{\prime}\right]}\right] \\
\simeq \frac{1}{\left(\alpha_{1}+\alpha_{2}\right)} \frac{1}{\left(\alpha_{1}+\beta_{1} \tau_{1}^{\prime}\right)},
\end{gathered}
$$

where we neglected small contributions proportional to infinitesimal mass. Therefore we obtain

$$
\begin{aligned}
I_{s} & =(2 \pi i)^{2} m^{2} \int_{0}^{\infty} d \beta_{2} \frac{1}{\left(1+\beta_{2}\right)^{2}} \int_{0}^{\infty} d \alpha_{1} d \alpha_{2} \frac{\theta\left(\alpha_{1}>m^{2}\right)}{\left(\alpha_{1}+\alpha_{2}\right)^{2}} \int_{0}^{\infty} d \beta_{1} \frac{\theta\left(\alpha_{2} \beta_{2}>m^{2}\right)}{\left(\beta_{1}+\tau_{1}^{\prime} \tau_{1}\right)} \frac{1}{\left(\alpha_{1}+\beta_{1}\right)}(132 \\
& =(2 \pi i)^{2} m^{2} \int_{m^{2}}^{\infty} d \alpha_{1} \frac{\ln \left[\alpha_{1} / \tau_{1}^{\prime} \tau_{1}\right]}{\alpha_{1}-\tau_{1}^{\prime} \tau_{1}} \int_{0}^{\infty} d \alpha_{2} \frac{1}{\left(\alpha_{1}+\alpha_{2}\right)^{2}\left(1+m^{2} / \alpha_{2}\right)} \\
& \simeq(2 \pi i)^{2} \int_{1}^{\infty} \frac{d \alpha_{1}}{\alpha_{1}}\left(\ln \alpha_{1}+\ln \left[m^{2} / \tau_{1}^{\prime} \tau_{1}\right]\right) \frac{\alpha_{1}-\ln \alpha_{1}-1}{\left(1-\alpha_{1}\right)^{2}} \\
& =(2 \pi i)^{2} \ln \left[\tau_{1}^{\prime} \tau_{1} / m^{2}\right]\left(1-\frac{\pi^{2}}{6}\right)+\mathcal{O}(1)
\end{aligned}
$$

Therefore we finally arrive at:

$$
I_{s}=(2 \pi i)^{2} \ln \left[\tau_{+} \tau_{-} / m^{2}\right]\left(1-\frac{\pi^{2}}{6}\right)+\mathcal{O}(1) .
$$




\section{Appendix E: Calculation of $\Psi_{20}^{\boldsymbol{\beta}}\left(k_{i}^{-}\right)$}

Let us rewrite the Eq.(76) as

$$
\Psi_{20}^{\boldsymbol{\beta}}\left(k_{i}^{-}\right)=\bar{\chi}_{\beta_{1}^{\prime} \beta_{2}^{\prime} \beta_{3}}^{\prime}\left[\gamma^{i}\right]_{\beta_{1}^{\prime} \beta_{1}}\left[\gamma^{i}\right]_{\beta_{2}^{\prime} \beta_{2}} J_{20},
$$

with

$$
\begin{aligned}
J_{20}= & \int d k_{1}^{+} d k_{2}^{+} d k_{1 \perp} d k_{2 \perp} \frac{m^{2}}{\left[k_{2}^{2}-m^{2}\right]\left[k_{1}^{2}-m^{2}\right]} \\
& \times \frac{-2\left(p^{\prime} k_{1}\right)}{\left[\left(p^{\prime}-k_{1}-k_{2}\right)^{2}-m^{2}\right]\left[\left(p^{\prime}-p_{3}^{\prime}-k_{1}\right)^{2}-m^{2}\right]\left(k_{1}+k_{2}-p^{\prime}+p_{3}^{\prime}\right)^{2}\left(k_{1}-p_{1}^{\prime}\right)^{2}} .
\end{aligned}
$$

The exact answer for $J_{20}$ is very complicated. We shall compute this expression only in the region of small $k_{i}^{-} \rightarrow 0$ assuming that their ratio is fixed $k_{1}^{-} / k_{2}^{-} \sim \mathcal{O}(1)$. Again, redefine for simplicity light-cone decomposition:

$$
k_{i}=k_{i}^{-} \frac{n}{2}+k_{i}^{+} \frac{\bar{n}}{2}+k_{i \perp} \equiv \alpha_{i} \frac{n}{2}+\beta_{i} \frac{\bar{n}}{2}+k_{i},
$$

and rewrite Eq.(138) as

$$
\begin{aligned}
J_{20} & =\int d \beta_{1} d \beta_{2} d k_{2} d k_{1} \frac{m^{2}}{\left[\alpha_{2} \beta_{2}-k_{2}^{2}-m^{2}\right]\left[\alpha_{1} \beta_{1}-k_{1}^{2}-m^{2}\right]} \\
& \times \frac{\left(-Q \beta_{1}\right)}{\left[\left(\alpha_{1}-\bar{y}_{3} Q\right) \beta_{1}-k_{1}^{2}\right]\left[\left(\alpha_{1}-y_{1} Q\right) \beta_{1}-k_{1}^{2}\right]} \\
& \times \frac{1}{\left[\left(\beta_{1}+\beta_{2}\right)\left(\alpha_{1}+\alpha_{2}-Q\right)-\left(k_{1}+k_{2}\right)^{2}\right]\left[\left(\beta_{1}+\beta_{2}\right)\left(\alpha_{1}+\alpha_{2}-\bar{y}_{3} Q\right)-\left(k_{1}+k_{2}\right)^{2}\right]} .
\end{aligned}
$$

Performing integrations over $\beta_{1}$ and $\beta_{2}$ by residues we can neglect by all poles for which

$$
\alpha_{i}>Q-\alpha_{j} \text { or } \alpha_{i}>Q y_{i}-\alpha_{j},
$$

because in this case $\alpha_{i}$ can not be small. Then we have contribution only from the poles associated with the first two propagators in Eq.(140) (in the first line). The result can be written as

$$
\begin{aligned}
J_{20} & \simeq(2 \pi i)^{2} \theta\left(0<\alpha_{1}<Q \alpha_{1}^{\max }\right) \theta\left(0<\alpha_{2}<Q \alpha_{2}^{\max }\right) \\
& \times \int d \beta_{1} d \beta_{2} d k_{2} d k_{1} \delta\left(\alpha_{2} \beta_{2}-k_{2}^{2}-m^{2}\right) \delta\left(\alpha_{1} \beta_{1}-k_{1}^{2}-m^{2}\right) \frac{\left(-Q \beta_{1}\right) m^{2}}{\text { Den }} .
\end{aligned}
$$

with the denominator

$$
\begin{array}{r}
\text { Den }=\left[\left(\alpha_{1}-\bar{y}_{3} Q\right) \beta_{1}-k_{1}^{2}\right]\left[\left(\alpha_{1}-y_{1} Q\right) \beta_{1}-k_{1}^{2}\right]\left[\left(\beta_{1}+\beta_{2}\right)\left(\alpha_{1}+\alpha_{2}-Q\right)-\left(k_{1}+k_{2}\right)^{2}\right] \\
\times\left[\left(\beta_{1}+\beta_{2}\right)\left(\alpha_{1}+\alpha_{2}-\bar{y}_{3} Q\right)-\left(k_{1}+k_{2}\right)^{2}\right]
\end{array}
$$

The maximal values $\alpha_{1}^{\max } \sim \alpha_{2}^{\max } \sim 1$. Their explicit values are not important. The integrand can be further simplified. We can neglect the small fractions $\alpha_{i} \ll Q$ in the denominator (143) that yields

$$
\begin{aligned}
& \text { Den } \simeq\left[\left(-\bar{y}_{3} Q\right) \beta_{1}-k_{1}^{2}\right] {\left[\left(-y_{1} Q\right) \beta_{1}-k_{1}^{2}\right] } \\
& \times\left[\left(\beta_{1}+\beta_{2}\right)(-Q)-\left(k_{1}+k_{2}\right)^{2}\right]\left[\left(\beta_{1}+\beta_{2}\right)\left(-\bar{y}_{3} Q\right)-\left(k_{1}+k_{2}\right)^{2}\right] .
\end{aligned}
$$

Then we take into account that the dominant contribution arises from the region where

$$
k_{i}^{2}=\alpha_{i} \beta_{i}-m^{2} \ll Q,
$$

as it follows from the $\delta$-functions in Eq.(142). Therefore we can also neglect the transverse momenta $k_{i}$ in the propagators in Eq.(144):

$$
\text { Den } \simeq\left[\left(-\bar{y}_{3} Q\right) \beta_{1}\right]\left[\left(-y_{1} Q\right) \beta_{1}\right] \frac{1}{\left[\left(\beta_{1}+\beta_{2}\right)(-Q)\right]\left[\left(\beta_{1}+\beta_{2}\right)\left(-\bar{y}_{3} Q\right)\right]}=\frac{\left(-m^{2}\right)}{y_{1} \bar{y}_{3}^{2} Q^{3}} \frac{1}{\beta_{1}\left(\beta_{1}+\beta_{2}\right)^{2}} .
$$


Finally we obtain

$$
\begin{aligned}
J_{20} \simeq & (2 \pi i)^{2} \theta\left(0<\alpha_{1}<Q \alpha_{1}^{\max }\right) \theta\left(0<\alpha_{2}<Q \alpha_{2}^{\max }\right) \frac{(-1)}{y_{1} \bar{y}_{3}^{2} Q^{3}} \\
& \times \int d \beta_{1} d \beta_{2} d k_{2} d k_{1} \delta\left(\alpha_{2} \beta_{2}-k_{2}^{2}-m^{2}\right) \delta\left(\alpha_{1} \beta_{1}-k_{1}^{2}-m^{2}\right) \frac{m^{2}}{\beta_{1}\left(\beta_{1}+\beta_{2}\right)^{2}} .
\end{aligned}
$$

A simple calculation yields:

$$
\begin{aligned}
J_{20} & \simeq(2 \pi i)^{2} \theta\left(0<\alpha_{1}<Q \alpha_{1}^{\max }\right) \theta\left(0<\alpha_{2}<Q \alpha_{2}^{\max }\right) \frac{(-1)}{y_{1} \bar{y}_{3}^{2} Q^{3}} \alpha_{2} \ln \left(1+\alpha_{1} / \alpha_{2}\right) \\
& \equiv(2 \pi i)^{2} \theta\left(0<k_{1}^{-} / Q<k_{1 \text { max }}^{-}\right) \theta\left(0<k_{2}^{-} / Q<k_{2 \max }^{-}\right) \frac{(-1)}{y_{1} \bar{y}_{3}^{2} Q^{3}} k_{2}^{-} \ln \left(1+k_{1}^{-} / k_{2}^{-}\right) .
\end{aligned}
$$

Substituting this into Eq.(137) we obtain the required result.

[1] N. Kivel and M. Vanderhaeghen, Phys. Rev. D 83 (2011) 093005 arXiv:1010.5314.

[2] M. K. Jones et al. [Jefferson Lab Hall A Collaboration], Phys. Rev. Lett. 84, 1398 (2000).

[3] V. Punjabi et al., Phys. Rev. C 71, 055202 (2005) [Erratum-ibid. C 71:069902 (2005)].

[4] O. Gayou et al. [Jefferson Lab Hall A Collaboration], Phys. Rev. Lett. 88, 092301 (2002).

[5] A. J. R. Puckett et al., Phys. Rev. Lett. 104, 242301 (2010).

[6] C. E. Hyde-Wright and K. de Jager, Ann. Rev. Nucl. Part. Sci. 54, 217 (2004).

[7] J. Arrington, C. D. Roberts and J. M. Zanotti, J. Phys. G 34, S23 (2007).

[8] C. F. Perdrisat, V. Punjabi and M. Vanderhaeghen, Prog. Part. Nucl. Phys. 59, 694 (2007).

[9] J. Arrington, K. de Jager and C. F. Perdrisat, arXiv:1102.2463.

[10] M. Sudol et al., Eur. Phys. J. A 44, 373 (2010).

[11] U. Wiedner, arXiv:1104.3961.

[12] A. Duncan and A. H. Mueller, Phys. Rev. D 21, 1636 (1980).

[13] A. Duncan and A. H. Mueller, Phys. Lett. B 90, 159 (1980).

[14] A. I. Milshtein and V. S. Fadin, Yad. Fiz. 33, 1391 (1981).

[15] A. I. Milshtein and V. S. Fadin, Yad. Fiz. 35, 1603 (1982).

[16] N. Isgur and C. H. Llewellyn Smith, Phys. Rev. Lett. 52, 1080 (1984).

[17] N. Isgur and C. H. Llewellyn Smith, Nucl. Phys. B 317, 526 (1989).

[18] N. Isgur and C. H. Llewellyn Smith, Phys. Lett. B 217, 535 (1989).

[19] B. L. Ioffe and A. V. Smilga, Nucl. Phys. B 216, 373 (1983).

[20] V. A. Nesterenko and A. V. Radyushkin, Phys. Lett. B 115, 410 (1982).

[21] V. M. Braun, A. Lenz, N. Mahnke and E. Stein, Phys. Rev. D 65, 074011 (2002).

[22] V. M. Braun, A. Lenz and M. Wittmann, Phys. Rev. D 73, 094019 (2006).

[23] A. V. Radyushkin, Phys. Rev. D 58 (1998) 114008 arXiv:hep-ph/9803316.

[24] M. Diehl, P. Kroll and C. Vogt, Eur. Phys. J. C 26, 567 (2003) arXiv:hep-ph/0206288.

[25] P. Kroll and A. Schafer, Eur. Phys. J. A 26, 89 (2005) arXiv:hep-ph/0505258.

[26] T. Feldmann and M. W. Y. Yip, Phys. Rev. D 85 (2012) 014035 [arXiv:1111.1844 [hep-ph]].

[27] C. W. Bauer, S. Fleming and M. E. Luke, Phys. Rev. D 63, 014006 (2000).

[28] C. W. Bauer, S. Fleming, D. Pirjol and I. W. Stewart, Phys. Rev. D 63, 114020 (2001).

[29] C. W. Bauer and I. W. Stewart, Phys. Lett. B 516, 134 (2001).

[30] C. W. Bauer, D. Pirjol and I. W. Stewart, Phys. Rev. D 65, 054022 (2002).

[31] M. Beneke, A. P. Chapovsky, M. Diehl and T. Feldmann, Nucl. Phys. B 643, 431 (2002).

[32] M. Beneke and T. Feldmann, Phys. Lett. B 553, 267 (2003).

[33] J. C. Collins and F. Hautmann, Phys. Lett. B 472, 129 (2000) arXiv:hep-ph/9908467].

[34] A. V. Manohar and I. W. Stewart, Phys. Rev. D 76, 074002 (2007) arXiv:hep-ph/0605001].

[35] V. L. Chernyak and A. R. Zhitnitsky, Phys. Rept. 112, 173 (1984).

[36] V. M. Braun, S. E. Derkachov, G. P. Korchemsky and A. N. Manashov, Nucl. Phys. B 553 (1999) 355 arXiv:hep-ph/9902375.

[37] V. M. Braun, G. P. Korchemsky and D. Mueller, Prog. Part. Nucl. Phys. 51 (2003) 311 arXiv:hep-ph/0306057.

[38] J. C. Collins and M. Diehl, Phys. Rev. D 61 (2000) 114015 arXiv:hep-ph/9907498.

[39] M. Beneke and V. A. Smirnov, Nucl. Phys. B 522 (1998) 321 arXiv:hep-ph/9711391], V. A. Smirnov and E. R. Rakhmetov, Theor. Math. Phys. 120 (1999) 870 [Teor. Mat. Fiz. 120 (1999) 64] arXiv:hep-ph/9812529], 
V. A. Smirnov, Phys. Lett. B 465 (1999) 226 arXiv:hep-ph/9907471, V. A. Smirnov, in Proc. of the 5th International Symposium on Radiative Corrections (RADCOR 2000) ed. Howard E. Haber, arXiv:hep-ph/0101152,

[40] V. A. Smirnov, Springer Tracts Mod. Phys. 177 (2002) 1.

[41] V. Y. Petrov and M. V. Polyakov, arXiv:hep-ph/0307077

[42] D. Diakonov, V. Y. Petrov and P. V. Pobylitsa, Nucl. Phys. B 306 (1988) 809.

[43] D. Diakonov and V. Y. Petrov, arXiv:hep-ph/0009006

[44] V. Y. Petrov, private communication.

[45] B. Jantzen, JHEP 1112 (2011) 076 arXiv:1111.2589 [hep-ph]].

[46] J. Y. Chiu, A. Fuhrer, A. H. Hoang, R. Kelley and A. V. Manohar, Phys. Rev. D 79 (2009) 053007 arXiv:0901.1332 [hep-ph]].

[47] A. Idilbi and T. Mehen, Phys. Rev. D 75, 114017 (2007) arXiv:hep-ph/0702022.

[48] J. C. Collins, Foundations of Perturbative QCD (Cam- bridge University Press, Cambridge, 2011).

[49] G. Bell and T. Feldmann, Nucl. Phys. Proc. Suppl. 164 (2007) 189 arXiv:hep-ph/0509347.

[50] B. O. Lange and M. Neubert, Nucl. Phys. B 690 (2004) 249 [Erratum-ibid. B 723 (2005) 201] arXiv:hep-ph/0311345.

[51] M. Beneke, Nucl. Phys. Proc. Suppl. 111 (2002) 62 hep-ph/0202056.

[52] T. Becher and M. Neubert, Eur. Phys. J. C 71 (2011) 1665 arXiv:1007.4005 [hep-ph]].

[53] T. Becher, G. Bell and M. Neubert, Phys. Lett. B 704 (2011) 276 arXiv:1104.4108 [hep-ph]].

[54] M. Garcia-Echevarria, A. Idilbi and I. Scimemi, arXiv:1111.4996 [hep-ph].

[55] J. Y. Chiu, A. Jain, D. Neill and I. Z. Rothstein, arXiv:1202.0814 [hep-ph].

[56] M. Beneke, G. Buchalla, M. Neubert and C. T. Sachrajda, Nucl. Phys. B 591 (2000) 313 arXiv:hep-ph/0006124.

[57] M. Beneke and T. Feldmann, Nucl. Phys. B 592 (2001) 3 arXiv:hep-ph/0008255. 